\title{
Nursing Abstracts from the 2nd Conference of the World Federation of Critical Care
}

\section{Nurses}

\section{RELATIONSHIP BETWEEN ORTHOSTATIC HYPOTENSION AND POSTPRANDIAL HYPOTENSION CL Lee ${ }^{1}$, SJ Tzou ${ }^{2}$}

1 National Defense Medical Center; 2 Kaoshiung Military General Hospital

\section{Objectives}

The purpose of this study was to examine the prevalence and relationship among the orthostatic hypotension $(\mathrm{OH})$, postprandial hypotension (PPH) and symptoms resulting from impaired perfusion of various organs. With increasing age, the $\mathrm{OH}$ occurring the elder group, assuming the upright position is more often accompanied by symptoms such frail, dizziness and ischemia of fetal organ (such as $\mathrm{MI}$ ), possibly damage to die. $\mathrm{OH}$ is a common clinical finding in older person, depending on the characteristics of the target population and the definition of the disorder. The previous report the prevalence of $7 \%$ in health and normal data of blood pressure (BP), 20\% occur on sickness and age over 65 years, increasing to $30 \%$ of age over 75 years old. According to the results of some epidemiological studies, orthostatic hypotension is associated with advanced age, whereas others have found no association.

Methods

The research collects the demography-matching sampling, the volunteers who can supine and continues over 3 minutes, from the general ward admitted over 65 years by the onsite blood pressure measurement and symptoms interview. The 60 responses, recording their blood pressure and heart beat during first minute and third minutes in supine and change to sit for six times, including general position, before and after the meal as lunch, control by the symptoms of supine and induced dizziness related medication usage.

Results

The prevalence was $1-3 \%$ as different as previous study review, especial occurring on post-standing 3 minutes of non-fasting phase. The differences of blood pressure existed most in post- breakfast 2 hours, post-lunch 2 hours than post-lunch period.

Conclusions

The results show many symptoms of impaired perfusion happened during clinical setting and difficult to differential between orthostatic hypotension, postprandial hypotension. The research team still suggested finding more predicted factor and correlation to prevent the impaired perfusion symptoms induced fall in the future research. The results will apply for the clinical health education to elder of ambulance safety, especial the critical time within 3 minutes when the client change position.

0010 ASSESSMENT OF THE NEAR DROWNING CHILD F Martens, J Ramet

Vrije Universiteit Brussels Belgium

\section{Background}

Drowning is the second most common cause of unintentional injury-related death among children less than 15 years old and is the leading cause of unintentional injury-related death in children from 1 to 4 years old.

Swimming pools account for the major near-drowning accidents, while bathtubs and water filled buckets are the second mostcommon site for preschool drownings.

\section{Objectives}

This presentation outlines the management of the near-drowning child. The principal physiological consequences of submersion incidents are hypoxia, ischemia, respiratory and metabolic acidosis and hypothermia. In most cases aspiration of liquid is involved which leads to a persistent impairment of the gas exchange. Arrhythmias and hypovolemia are very likely to occur. The main objective of treatment of the near-drowned patient is limiting neurological damage.

\section{Discussion}

Cardiopulmonary resuscitation is the mainstay of immediate management. Aggressiveness and success of the initial resuscitation are strong determinants of long-term prognosis. Initiation of basic and advanced paediatric life support are of vital importance. Treatment consists of resuscitation and stabilization following the European Paediatric Life Support guidelines.

\section{Conclusions}

The prognosis after a submersion accident depends in the first place on the duration of the submersion, which is often difficult to establish. Submersion for over 5 minutes is prognostically unfavourable. In hypothermia due to submersion the prognostic factors are less clear, in these cases the treatment should always be continued until the core temperature is $32^{\circ} \mathrm{C}$ or higher. Death from cardiac arrest in hypothermia is failure to respond to resuscitation and rewarming. You are not dead until you are warm and dead.

\section{O029 COMPARISON OF NURSES AND PATIENTS ASSESS-} MENT OF SLEEP IN CRITICAL CARE

A Richardson ${ }^{1}$, W Crow ${ }^{1}$, E Coghill ${ }^{1}$, C Turnock $^{2}$

1 Newcastle upon Tyne Hospitals;2 Northumbria University

\section{Background}

Sleep is important for promoting critical care patient recovery and sleep disturbance is known to cause irritability, aggression and increased stress levels. Despite the importance of sleep, nurses don't acknowledge this when planning patient care. Objective and subjective ways to measure patients sleep have been researched but within critical care the availability and use of valid critical care sleep assessment tools is limited. 


\begin{abstract}
Objectives
To compare nurses' assessment of patients sleep with patients' own assessment of their sleep using three user-friendly non-interventional sleep assessment tools in critical care.

\section{Methods}

Three sleep assessment-rating scales were constructed to provide easy to understand tools for completion by both patients and nurses in critical care. 82 patients and 82 nurses participated in the study using a convenience sample from four multi-specialty critical care units in one large teaching trust. Patients were included in the study if they met a list a criteria to obtain the most appropriate responses from lucid orientated critically ill patients.
\end{abstract}

\section{Results}

No tool produced a close association between the nurses' assessment of the patients sleep and the patients' assessment of their sleep. Patients found two of the three tools easy to use when rating their sleep.

\section{Discussion}

Objective invasive measurements of sleep as well as complex subjective tools appear inappropriate to be used as a part of daily critical care practice. The application of simple rating scores has a high degree of error when nurses assess patients' sleep, even though high levels of patient observation and assessment are practised in critical care.

\section{Conclusions}

More research is needed to examine the assessment of patients sleep in critical care, particularly linking rating scales to alternative methods of physical assessment and the assessment of sleep over the complete 24-hour period.

\section{O030 EVALUATING THE IMPACT OF 12 HOUR SHIFTS IN CRITICAL CARE}

A Richardson 1, C Turnock², A Finley1, L Harris 1, S Carson1

1 Newcastle upon Tyne Hospitals NHS trust;2 Northumbria University

\section{Background}

Emphasis has been placed upon improving the working lives of staff within the NHS and 12-hour shifts has shown to be one approach contributing to a more flexible pattern of work. Most studies have been conducted on staff working in non-critical care settings and have found varying effects on continuity of care, fatigue and flexible working patterns.

\section{Objectives}

The study aims were to examine the impact of 12-hour shifts on critical care staff and analyse the implications of 12-hour shifts for the working practices within critical care settings.

\section{Methods}

Data was collected using a staged dual approach consisting of two focus groups with 16 critical care nurses and a questionnaire survey to a sample of $192,77 \%$ of whom returned questionnaires from four critical care units in Northeast of England. Focus group data were transcribed and analysed to identify themes and questionnaire data were analysed using SPSS.

\section{Results}

The main findings of the study included the following. The majority of Critical Care nurses perceived 12 shifts as having a positive impact on their ease to plan and prioritise care throughout a shift and with the allocation of sufficient time for nurse-nurse handover. Most nurses found 12-hour shifts lead to improved patient and family relationships. However mixed views were found with the impact of 12 hour shifts on motivation and tiredness at work. A high proportion of nurses working 12 hour shifts found this led to good quality time off work, easier and cheaper to travel to work and made off duty requests more likely to be met. $86 \%$ of staff thought the 12-hour shift system should continue and areas for improvement were suggested.

\section{Conclusion}

Despite the limitations, this study found common issues with the effects of 12-hour shifts in critical care settings and previous research. If 12-hour shift systems enable flexible working practices, strategies should be established to minimise fatigue and tiredness on staff in the future.

\section{O042 THE CURRENT STATUS OF FAMILY BEREAVEMENT PROGRAMS IN AUSTRALIAN INTENSIVE CARE UNITS}

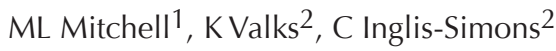

1 Griffith University;2 Princess Alexandra Hospital

\section{Objectives}

The aim of the study was to identify the prevalence and type of bereavement services offered to families of critically ill patients in Australian Intensive Care Units.

\section{Methods}

Nurse unit mangers from adult Intensive Care Units throughout Australia were contacted by telephone and invited to participate. A twenty-item survey was mailed to them to complete. Returned survey data were entered into Statistical Package for Social Sciences for collation of descriptive statistics. Open-ended questions were transcribed in full and coded by searching for similarities and differences.

\section{Results}

One hundred and seventeen Intensive Care Units were contacted and 99 completed questionnaires. Most units offer fundamental components of bereavement programs such as viewing of the deceased and communicating with family members. Less than one third $(n=26)$ provide additional follow-up services. Program evaluation is primarily achieved through verbal feedback from staff and families with only two units using a formal evaluation of families about their program. Over half of the respondents indicated they are considering or interested in providing a bereavement program.

\section{Conclusions}

This study highlights the need for research-based data to support interventions for bereavement programs using family-centred outcome measures. Importantly, current bereavement programs need a more rigorous method of evaluation. Intensive Care nurses are interested in this area of clinical practice and require considerable support. It is recommended that this support occurs through postgraduate and on-going education, hospital policies and procedures. 


\section{* Nursing Abstracts from the 2nd Conference of the World Federation of Critical Care Nurses}

\section{O043 IMPROVING TRANSFER FROM INTENSIVE CARE FOR FAMILIES AND NURSES BY USING A STRUCTURED INDIVIDUALIZED FORMAT}

ML Mitchell

Griffith University

\section{Background}

The intervention study examined the efficacy of a structured individualised method of patient transfer from Intensive Care to general wards from the perspective of families and Intensive Care Unit nurses. A specifically designed brochure provided nurses with key points to discuss with families whose relative was for transfer.

\section{Methods}

A mixed method design was used. The researcher purposively allocated family members to a control $(n=80)$ and intervention group $(n=82)$. The intervention group experienced a structured individualised transfer method whereas the control group received existing ad hoc transfer methods. Families were surveyed after transfer with a ten-item questionnaire. Intensive Care Unit nurses were also surveyed on their perceptions of the usefulness of the structured transfer brochure.

\section{Results}

Those family members who experienced the formal transfer reported significantly higher levels of satisfaction with the information given to them before transfer from Intensive Care than did the control group $(p=.015)$. The intervention group also recorded significantly higher scores when their level of understanding of information was evaluated $(p=.002)$ and they felt significantly more prepared for transfer than those in the control group $(p<.001)$.

The Intensive Care Unit nurses $(n=33)$ considered that the structured intervention assisted them by supporting and directing their discussion about patient transfer with family members. $95 \%$ of respondents indicated it provided a useful framework for them to use and recommended its introduction for all patient transfers from Intensive Care.

\section{Conclusions}

This study supports the introduction of a two-tiered approach to sharing information with family members prior to transfer. The faceto-face communication and individualised brochure for the family contributed significantly to families' satisfaction, preparation and communication about patient transfer and is thus recommended. The Intensive Care Unit nurses felt supported by the brochure in their role of transferring patients and recommend its introduction for all patient transfers.

\section{0}

\section{KNOWLEDGE OF THE NURSING PROFESSIONALS CONCERNING THE ATTENDANCE TO THE CHILDREN WITH STOPPED CARDIO-RESPIRATORY}

RMC Coutinho, EMS Amaral

Paulista University, São Paulo, Brazil

\section{Background}

In pediatrics, the event of a cardio-respiratory stop normally occurs as resulted of the acute respiratory insufficiency and/or cardiovascular shock. The recognition of the basic cause can contribute in the immediate therapeutically decisions, diminishing the risk of complications for measures, to the times, unnecessary. In situation of cardio-respiratory stop the team must act fast and correctly to recoup the child, being priority the identification of the problems and the precocious intervention with a qualified assistance, providing an efficient treatment. The assistance of nursing systemize for the nurse in the cardio-respiratory stop reduces stress of the team, better rationalizes the time of the cares, gets greater participation of the nursing team and prevents material wastefulness.

\section{Objectives}

To evaluate the knowledge of the team of nursing concerning the assistance to the child in situation of cardio-respiratory stop.

\section{Methods}

It is a quantitative; explore study, which used a composed questionnaire for 12 structuralized questions and 2 half-structuralized that it approached the knowledge of the nursing team, front to the attendance of cases of cardio-respiratory stop in children. The sample was composed for 28 individuals that if had fit to the definite criteria and if they had made use to answer.

\section{Results}

When analyzing the answers we got that the nurse aid had made right $49.44 \%$ of the questions, what means the half of the questions; the nursing technician that they had presented a superior knowledge of the excessively professional ones of the sector, them had made right $74.07 \%$ of the questions; while that the nurses had made right $62.22 \%$ of the questions. When relating the answers of all professionals of nursing we find $54.39 \%$ of rightness, little more of the half of the questions. The subjects with more assertive answers in the cardio-respiratory stop in children had been: signals and symptoms, after diagnostic immediate behavior, material resources, managed medicines, relevance of the registers of the nursing notations and ways of administration of medicines. The subjects with errs answers in the cardio-respiratory stop in children had been: corporal position to carry through the external thorax compression, positioning of the hands for accomplishment of the thorax compression and purpose of the drugs. These results indicate that it has necessity of the accomplishment of an improvement to prevent complications due the lack of specific knowledge for this attendance. Conclusions

The data gotten in this research in allow them to conclude that most $71.43 \%$ of the professionals is nurse aid and the nursing team made right the majority of the referring questions to the cardio-respiratory stop in children. One becomes essential that the hospital invests in the team and organizes a program of qualification and update in emergency so that the nursing professionals can be always prepared to give assistance to the cardio-respiratory stop in children, contributing of efficient form for its reestablishment.

\section{O059 HONG KONG PUBLIC AWARENESS OF CORONARY HEART DISEASE}

CW Chan, V Lopez, DR Thompson

The Nethersole School of Nursing, The Chinese University of Hong Kong

\section{Background}

Coronary heart disease (CHD) has been a major cause of death and disability. The World Health Organisation reports that risk factors of CHD causes millions of deaths per year, $75 \%$ of deaths was related to cardiovascular disease. In Hong Kong, CHD is the second leading cause of death since the 1960s. Reduction of CHD risks, prevention of the disease and promoting health are viewed as global concerning issues. While much is known about lay pub- 
lic's perspectives about CHD among the western cultures, there remains a paucity of knowledge about $\mathrm{CHD}$ from the perspective of the Chinese people.

\section{Objectives}

A descriptive qualitative research design was used to explore Hong Kong Chinese public understanding of CHD using tape-recorded focus group interviews.

\section{Methods}

Twelve focus group interviews were conducted consisting of a total of 84 participants. All interviewees were tape recorded lasting from 60 to 90 minutes. All interviews were transcribed verbatim and content analysis was used to analyse the data.

\section{Results}

A number of key categories about CHD were identified. These included misconceptions of CHD as a disease of the blood vessel, varying descriptions and representations of $\mathrm{CHD}$ symptom, $\mathrm{CHD}$ as an age-related and gender-related disease, inappropriate lifestyle, and $\mathrm{CHD}$ as a disease of lesser concern compared to severe acute respiratory syndrome or cancer.

\section{Conclusions}

Despite differences in people's understanding about CHD, the results highlighted that great efforts in targeting measures based on various levels of people's understanding about CHD remain important. As coronary heart disease requires an individual to adhere to preventive advice and lifestyle changes, the individual understanding of $\mathrm{CHD}$ is central and should be closely examined.

\section{HONG KONG CHINESE PATIENTS' DECISION- MAKING PROCESS IN SEEKING EARLY TREATMENT FOR CHEST PAIN}

R Kaur ${ }^{1}$, V Lopez ${ }^{2}$, DR Thompson ${ }^{2}$

1 Hong Kong Adventist Hospital;2 The Chinese University of Hong Kong

\section{Background}

Chest pain is a major cause of morbidity and mortality, often indicative of angina or acute myocardial infarction (AMI). The importance of prompt institution of reperfusion therapy has led to public education to reduce time between onset of symptoms and treatment. A review of patients with chest pain showed a medium delay time ranging from two to seven

hours. The causes of delay are multidimensional with most research focusing on socio-demographic, healthcare system and situational factors.

\section{Objectives}

The objective of this study was to explore the factors that influence Hong Kong Chinese patients' decision-making in seeking early treatment upon the onset of chest pain.

\section{Methods}

A descriptive qualitative study using in-depth tape-recorded interviewing technique was conducted over six months. Purposive sample was employed and included only those participants who met the inclusion criteria: diagnosis of chest pain indicative of AMI or unstable angina; stable 24 hours after admission; ethnic Chinese living in Hong Kong; and over 18 years of age. Data was analysed using content analysis.

\section{Results}

Twenty seven (11 female and 16 male) participants were interviewed. The age ranged from 41 to 82 years (mean $=65, S D=11$ ). The mean time from the onset of symptoms to triage was 925.52 $(\mathrm{SD}=1252.35)$ minutes for men and $3232.86(\mathrm{SD}=2839.88)$ for women. The delay time for the Hong Kong participants was far longer compared to the western population with an average of two to six hours. From the qualitative data, three categories were discerned: (1) becoming aware of the threat; (2) maintaining a sense of normality; and (3) struggling to mobilize resources.

\section{Conclusions}

The findings indicate that Hong Kong Chinese patients delay seeking treatment far more longer than the western counterparts. A variety of decisions were made by patients from the onset of chest pain to seeking help. The findings also indicate that their decisions to seek help were heavily guided by their cultural beliefs and values and a lack of cognitive representation of the symptoms as serious threat, thus prolonging delay.

\section{BEDSIDE CHECKLIST FOR NURSES, QUALITY ASSURANCE IN MANAGEMENT OF CRITICALLY ILL PATIENTS}

K Wong, M O'Hehir, A Driver

Monash Medical Centre, Clayton, Victoria, Australia

\section{Background}

Monash Intensive Care unit is a combined adult and paediatric unit, admitting patients suffering from a wide range of health problems requiring critical care management. The nursing workforce is comprised of nurses with varying educational levels, knowledge and experience in the critical care area, ranging from Clinical Specialists to graduate nurses and transient agency nurses. In order to coordinate patient quality care within the unit, the nursing management committee has designed a bedside checklist.

The aim of implementing this checklist is to ensure patient and staff safety around the bed area while alerting nursing staff to the importance of clinical observations and physical examination of their patients. After a 3 month trial of the checklist, the nursing management committee conducted a survey of the Bedside Checklist among nurses working in the unit.

\section{Objectives}

1. To study the critical care nurses' impressions about the Bedside Checklist.

2. To establish a basic safety checklist model to enhance the patient quality management.

\section{Methods}

The survey was conducted among a group of nurses who were working in one-single intensive care unit. The study subjects were recruited in a convenience sampling method. The questionnaires enclosed in the survey included respondents' demographic data, a combination of a Likert Scale designed quantitative questions and qualitative open-ended questions. These questions were designed to seek nurses' impressions about the checklist design and the effective use of the Bedside Checklist.

\section{Results}

There were 110 surveys delivered to nurses. The number of respondents to the survey was 65 . The results show that majorities of staff agree or most likely agree that: 1 . the Bedside Checklist is a well 


\section{Nursing Abstracts from the 2nd Conference of the World Federation of Critical Care Nurses:}

designed systematic guideline for nursing professionals (odds ratio: $3.31)$; 2. following the Bedside Checklist is a good clinical reflective practice to nurses (odds ratio: 2.00); 3. all items listed in the Bedside Checklist are essential criteria to delivering safe patient care (odds ratio: 2.43 ); 4 . mandatory compliance to the bedside checklist is a good mechanism for quality management of patients (odds ration: 1.5). With sub-group studies, $50 \%$ of the first year graduated nurses and the critical care course students agree or most likely agree that mandatory utilisation of the Bedside Checklist will improve patient care standard.

\section{Conclusions}

As professional critical care nurses, we should maximise patient safety in the complex work environment and the Bedside Checklist for Nurses is a reliable method of detecting patient problems before any further determinations are made. The Bedside Checklist is a formal and contextual knowledge tool stating the responsibilities to patients' designated nurses. It is a contractual binding agreement between a nurse and his/her professionalism. It is a useful instrument for patient quality management that also has the potential to improve patients' quality of care.

\section{A PROSPECTIVE EVALUATION OF NURSE-DIRECT- ED PROTOCOLIZED WEANING FROM MECHANICAL VENTILATION ON PATIENT OUTCOMES IN THE UNITED KINGDOM}

B Blackwood $^{1}$, J Wilson-Barnett ${ }^{2}$, GG Lavery ${ }^{3}$

1 Queen's University Belfast, Belfast, Northern Ireland;2 King's College London, London, England;3 Royal Group of Hospitals Trust, Belfast, Northern Ireland

\section{Background}

Growing international evidence demonstrates that respiratorytherapist or nurse-directed protocols for weaning from mechanical ventilation $(\mathrm{MV})$ are safe and efficient and may lead to a reduction in MV time when compared with traditional weaning methods. Since the Department of Health established the Modernisation Agency Critical Care Programme in the United Kingdom (UK) in 2000, several intensive care units (ICUs) introduced nurse-directed weaning: however its efficacy in the UK health care system had not been evaluated.

\section{Objectives}

To compare nurse-directed protocolised weaning with traditional doctor-led weaning practice.

\section{Methods}

A non-randomised comparative trial conducted in $2 \mathrm{ICU}$ in 2 university-affiliated teaching hospitals from January 2001 to December 2003. The sample comprised all patients receiving MV. Outcome measures (MV time, intubation time, ICU stay, and mortality and reintubation rates) were collected before (phase 1) and after (phase 2) the intervention in both units.

\section{Results}

After implementing nurse-directed weaning the geometric means (GM) for MV time, intubation time and ICU stay increased from baseline in both units. Regression-based analyses showed that changes from baseline were not significantly different between the 2 units for MV time $(p=0.37)$ and intubation time $(p=0.39)$ but were for ICU stay $(p=0.03)$. APACHE II and the patient's diagnostic category were significant predictors. Following adjustment for these covariates, changes from baseline in the intervention unit were not significantly different from changes in the control unit. The ratio of GMs phase 2:phase $1(95 \% \mathrm{Cl})$ before and after adjustment for APACHE II and diagnostic group are presented. Reintubation and mortality rates were not significantly different from baseline within both units.

\begin{tabular}{|l|l|l|l|l|}
\hline & $\begin{array}{l}\text { Phase 1 GM } \\
\text { (hours) }\end{array}$ & $\begin{array}{l}\text { Phase 2 GM } \\
\text { (hours) }\end{array}$ & $\begin{array}{l}\text { Ratio } \\
(95 \% \mathrm{Cl})\end{array}$ & $\begin{array}{l}\text { Ratio (adjusted) } \\
(95 \% \mathrm{Cl}) \\
\text { Control Unit }\end{array}$ \\
\hline $\mathrm{n}=134$ & $\mathrm{n}=117$ & & & MV time \\
\hline 105 & 132 & $1.26(0.90-1.76)$ & $1.18(0.84-1.67)$ & Intubation time \\
\hline 124 & 169 & $1.36(0.99-1.87)$ & $1.28(0.93-1.76)$ & ICU stay \\
\hline 200 & 212 & $1.06(0.83-1.37)$ & $1.03(0.79-1.34)$ & Intervention Unit \\
\hline $\mathrm{n}=197$ & $\mathrm{n}=214$ & & & MV time \\
\hline 86 & 131 & $1.53(1.17-2.00)$ & $1.21(0.94-1.55)$ & Intubation time \\
\hline 87 & 143 & $1.64(1.25-2.15)$ & $1.26(0.98-1.61)$ & ICU stay \\
\hline 123 & 193 & $1.57(1.26-1.96)$ & $1.33(1.08-1.63)$ & \\
\hline
\end{tabular}

\section{Conclusions}

In the UK, patient outcomes are not significantly affected when patients are weaned using nurse-directed protocols. This study highlights the key role of nurses in providing safe and efficient weaning of patients from MV. The findings support other studies advocating the efficiency of protocolised weaning.

\section{THE VALUE OF THE "RESEARCH COORDINATOR POSITION" IN AUSTRALIAN AND NEW ZEALAND INTENSIVE CARE UNITS}

BL Roberts ${ }^{1}$, CM Rickard ${ }^{2}$, JW Foote ${ }^{3}$, MR Mcgrail $^{4}$

1 Sir Charles Gairdner Hospital, Perth, Western Australia, Australia;2 Monash University, Latrobe Regional Hospital, Traralgon, Victoria, Australia;3 The Queen Elizabeth Hospital, Adelaide, South Australia, Australia;4 Monash University, Traralgon, Victoria, Australia

\section{Background}

The Research Coordinator $(\mathrm{RC})$ role is a relatively new addition to staffing profiles in Australasian Intensive Care Units (ICUs). The position was developed in the mid 1990s in response to the increasing quantity and complexity of pharmaceutical oriented research, the establishment of the Australia and New Zealand Intensive Care Society Clinical Trials Group (ANZICS CTG), and a growing interest in research and evidence based practice in the health professions. There have been anecdotal reports of RC positions in various speciality areas in the past. However, limited research has been undertaken into the role, and no study has been published in the ICU setting.

\section{Objectives}

1. Describe the demographics of Australasian ICU RCs (e.g. age, gender, education, professional experience). 2. Describe the structure and function of the RC role in Australasian ICUs (e.g. attributes of the position, type of research undertaken).

\section{Design \\ Cross-sectional cohort study.}

\section{Method}

After obtaining institutional ethics approval all members $(n=69)$ of the Australia and New Zealand Intensive Care Research Coordinators' Interest Group (IRCIG) were invited to participate. RCs completed a self-report anonymous questionnaire with regards to demographics and role structure using a secure website. The demographic data and open-ended responses for the best and worst 
aspects about the RC role were descriptively analysed using an adapted thematic method.

\section{Results}

49 participants $(71 \%)$ completed the survey. The majority were female aged between 31 and 50 years, who had worked in clinical $>9$ years. All respondents held tertiary qualifications with more than half $(57 \%)$ having postgraduate degrees. Nearly half the RCs had been in the position 1 to 3 years, with $2 \%>9$ years. Sponsored pharmaceutical trials accounted for the majority of work, followed by ANZICS CTG trials, departmental research, audits and personal research. Four thematic clusters for best and worst aspects of the job emerged: Job structure, job worth, work description and colleagues. The best aspects of the role as determined by the survey were autonomy and flexible hours (job structure); respect (job worth); intellectual stimulation (work description) and working in a multidisciplinary team (colleagues). The worst aspects of the role according to the survey were isolation and onerous on-call (job structure); being under-valued (job worth); excessive workload (work description) and lack of support from nursing management (colleagues).

\section{Conclusions}

The Australasian RC is usually an experienced nurse who values autonomy, respect and intellectual stimulation and finds isolation, workload and under-recognition to be the worst aspects of the position.

The results from this survey should assist management in finding ways to stimulate retention and attract the best staff for this valuable position.

\section{SATISFACTION WITH AND IMPORTANCE OF JOB VARIABLES FOR RESEARCH COORDINATORS IN AUS- TRALIAN AND NEW ZEALAND INTENSIVE CARE UNITS}

BL Roberts ${ }^{1}$, CM Rickard ${ }^{2}$, JW Foote ${ }^{3}$, MR Mcgrail $^{4}$

1 Sir Charles Gairdner Hospital, Perth, Western Australia, Australia;2 Monash University, Latrobe Regional Hospital, Traralgon, Victoria, Australia;3 The Queen Elizabeth Hospital, Adelaide, South Australia, Australia;4 Monash University, Traralgon, Victoria, Australia

\section{Background}

The pharmaceutical industry, many hospital research departments and Intensive Care Units (ICU) have recognized that optimal research management requires the employment of a research coordinator (RC). The RC plays a pivotal role in conducting ethical and scientifically sound research by adhering to universally recognized clinical research principles. Consistent with these requirements it is necessary to ascertain the significant factors of this unique role and to create job satisfaction in order to employ and retain the most suitable personnel.

\section{Objectives}

1. Measure RC job satisfaction

2. Establish what factors the $\mathrm{RC}$ values as important Design Cross-sectional cohort study

\section{Methods}

Following institutional ethics approval all members $(n=69)$ of the Australia and New Zealand Intensive Care Research Coordinators' Interest Group were invited to complete an anonymous questionnaire using a secure website. The questionnaire contained two parts with 31 questions each: (1) McCloskey-Mueller Satisfaction Scale (MMSS) and (2) MMSS-Importance Scale (MMSS-IS). Each part addressed job structure in thirteen questions, interaction with colleagues in ten and four questions each on job worth and work description. A Likert scale of 1 to 5 was used to score satisfaction/ importance with 1 representing the least and 5 the greatest satisfaction/importance. Tables of high/low importance versus high/low satisfaction were analyzed using Wilcoxon signed-rank test to allow prioritization for role development.

\section{Results}

$71 \%(n=49)$ participants completed the survey. SATISFACTION: 13 questions with themes such as working business hours, flexibility, level of responsibility and immediate colleagues scored high satisfaction (mean $>4$ ). 3 questions relating to childcare and compensation for weekends scored low (mean $<3$ ). Working hours, professional recognition and compensation for weekend work had the biggest range of high/low replies. IMPORTANCE: 22 questions on importance had mean scores $>4$ with themes of autonomy and professional relationship with peers being the most important. 9 questions scored mean $<4$ and $\geq 3$ with the lowest being availability of childcare facilities and social engagements with colleagues outside work. The biggest span of replies related to childcare. The largest discrepancies (mean difference $p<0.001$ ) where satisfaction did not meet importance included issues of salary, hours worked, recognition, career promotion and research processes.

\section{Conclusions}

The Australasian RCs are generally satisfied with the flexibility of the work and with their immediate colleagues. They find autonomy and their co-workers of greatest importance, but are dissatisfied with weekend work, salary, lack of professional recognition and limited career opportunities. A consensus on career and pay structure for RCs may improve their work conditions and promote professional recognition.

\section{IS IT POSSIBLE TO ORGANIZE POWERNAPPING DURING NIGHT SHIFT IN AN ICU?}

AT Jepsen, L Damgaard

Aarhus Sygehus, Aarhus, Denmark

\section{Background/Objectives}

Night shifts are an unavoidable fact when working in an ICU. Nurses complaining of various problems and symptoms in connection with night shifts made us realize that changes had to be done for nurses to function better in night shifts. It was necessary to ensure keeping the existing staff as well as to be able to recruit new nurses to the ward. Complaints from nurses in night shifts were characterized by feelings of fatigue between 3-6 am, reduced sleep quality causing increased use of sleeping medication, disrupted circadian rhythm, digestive trouble and reduced performance efficiency.

The ward consists of 6 intensive care stations, 50 staff members. Some nurses are working in 3 shifts and some are working night, evening or dayshifts only. A night shift lasts 8,5 hour. The average numbers of night shifts per person are 4-5 during 4 weeks. The nightshifts are manned by 4 registered nurses (one having the cooperative function), 1 nurse-assistant and 1 medical student (temporary substitute).

\section{Methods}

All staff members doing nightshifts were included in the powernapping project, which were ongoing during 3 month in the summer 


\section{* Nursing Abstracts from the 2nd Conference of the World Federation of Critical Care Nurses:}

of 2002. The length of the nap were planned to be 29 minutes - a break already included in the night shift and paid for by the employee. Consideration was made as to when to schedule the nap. In that respect the nurses own opinions and the complains made about night shifts were used. Furthermore practical nursing procedures during the night shift had to be evaluated as well as where to powernap and how to nap. The nurse in charge during the nightshift was responsible for co-ordinating the powernap and register problems in getting through the powernapping period.

\section{Results}

Powernapping was not accomplished 7 nights during the 3 month project period and 14 individuals were registered as not being able to powernap in particular nights. All staff members no mattes degree, were able to powernap. The individuals showed a great responsibility in organizing and planning the night shift in order to be able to powernap. Special nursing procedures were transferred to either the day or the evening shift without any consequences for the quality of the nursing. The powernapping location should be close to the ward. The powernap was scheduled from 3-6 am. Feedback concerning the length of the powernap (29 minutes) was positive. After powernapping during night shifts all staff involved reported increased wellbeing, reduced tiredness, acceptable sleeping rhythm (insomnia not reported as a side effect) less digestive problems, better performance efficiency and a feeling of the night shift being much shorter.

\section{Conclusions}

Powernapping is indeed possible to organize effectively provided each individual nurse show responsibility and give priority to the issue of powernapping. Another factor necessary is a practical and organized system for the project to work. Today powernapping is a routine in our ICU.

\section{E-LEARNING (ELECTRONIC-LEARNING) AS A METH- OD IN THE TRAINING OF NURSING STAFF AT AN ICU}

L Kjaer

Aarhus University Hospital

\section{Background/Objectives}

E-learning is a highly useful and effective method to guide and instruct in specific nursing procedures or in assembling of technical equipment. In all intensive care units, there is a huge assignment in educating and guiding new and experienced staff. For the newly arrived there is a tremendous amount of new equipment and new procedures to learn. For the experienced nurses there is continuously new equipment and new procedures to get acquainted with. Some procedures can be difficult to remember because they are complicated or only used rarely. It can be difficult to keep abreast of it all. Most units have books and briefcases with instructions and directions but often they can be difficult and time-consuming to use. Therefore we have developed a new learning- and updating method, Intensive E-learning, a computer program with short and clear instructions and directions that is available to all staff 24 hours a day.

\section{Methods}

Intensive E-learning is developed on a personnel computer and it has been an objective that the method is simple and easy to work with and to extend and further develop. Intensive E-learning has been elaborated as follows:

1. With a digital camera a series of pictures has been taken to illus- trate how to assemble technical equipment or to illustrate a nursing procedure step by step. 2 . The series of pictures are subsequently edited in a photo editing program, so they appear in a web-format where they take up as little space on the computer as possible and also ensure a faster execution for the users. 3. The series of pictures are set up in a PowerPoint show and afterward short, relevant and pedagogical instructions are added to the pictures in small textboxes. 4 . The series are linked together by means of an html-page: FrontPage.

\section{Results and Conclusions}

There is developed a system, Intensive E-learning, which consists of several interactive series of pictures, that is installed on personal computers next to the patients which are immediately available for the staff at all time. Intensive E-learning gives easy, educational and clear directions of different nursing procedures and instructions on how to assemble technical equipment. The advantage of Intensive E-learning is that the intensive Care Unit obtains a higher level of quality assurance in favour of the patients. For the staff the directions and instructions are easier to find and use than manuals in books and briefcases because pictures often illustrate better than the written language how equipment is assembled or procedures executed. The staff is this way given an alternative option for quick directions and instructions during the daytime but also during evening- and nightshifts where colleagues to ask for advice is less available. In spare time the staff has the possibility of clicking through the series and revive rare and complicated procedures.

\section{PATIENTS' DREAMS AND UNREAL EXPERIENCES IN ICU AS RECALLED TWO YEARS AFTER DISCHARGE: COMPARISON WITH DELIRIUM STATUS DURING AD-} MISSION

BL Roberts ${ }^{1}$, CM Rickard ${ }^{2}$, D Rajbhandari ${ }^{3}$, P Reynolds ${ }^{2}$

1 Sir Charles Gairdner Hospital, Perth, Western Australia, Australia;2 Monash University, Latrobe Regional Hospital, Traralgon, Victoria, Australia;3 Royal Prince Alfred Hospital, Sydney, New South Wales, Australia

\section{Background}

Intensive Care Unit (ICU) patients, whether agitated or lethargic, may experience vivid dreams. These may be persecutory in nature and frightening for the patients. Routine screening for delirium in ICU is becoming more prevalent, however, there has been little research comparing the objective development of delirium using screening tools and the patients' subjective recollection of dreams in the ICU.

\section{Objectives}

To describe (1) the patients' subjective experiences of dreaming during ICU admission and (2) any relationship between these dreams and the objective presence of delirium during that admission.

\section{Methods}

Following institutional ethics approval we conducted a prospective cohort study using the interview technique. The cohort was assembled from 152 patients who participated in a previously conducted multi-centre study of delirium incidence in Australian ICUs. The interviews involved a mixture of closed- and open-ended questions. The open-ended questions were a combination of descriptions and the use of semantic differential techniques relating to the ICU admission and dreams. To standardize the technique the same 
researcher at each site undertook the interview after joint training. Qualitative responses regarding recalled dreams and memories were analyzed using a thematic method. Demographic data was aggregated to allow description of the sample. Categorical data was compared using chi-square or Fisher's exact test, and continuous data was assessed for normality of distribution then compared using the t-test or Mann-Whitney $U$. Multivariate analysis was undertaken using logistic regression. A p-value of $<0.05$ was considered significant for all analyses.

\section{Results}

Review of hospital databases identified 103 patients potentially contactable 18 to 24 months after ICU discharge. 41 of these patients consented to participate and of these 18 patients (44\%) had been delirious and 23 patients (56\%) non-delirious during the ICU admission. Overall $44 \%$ of patients $(n=18)$ recalled dreams from their ICU admission. There was a trend to increased prevalence of dreaming (50\%) amongst the 18 delirious patients than for the 23 non-delirious patients (39\%), although this did not reach statistical significance (OR 1.56, 95\% $\mathrm{Cl} 0.45-5.41, \mathrm{p}=0.49$ ). Dreaming was significantly associated on multivariate modelling with increased length of stay (OR 1.39, 95\% $\mathrm{Cl} 1.08-1.79, \mathrm{p}=0.01$ ).

11 patients reported their dreams as scary and 10 patients describing good or indifferent dreams allowing for an overlap of 3 patients who experienced both.

\section{Conclusions}

It is important to provide optimal holistic care for ICU patients to restore both physical and psychological health and well being. The prevalence of $44 \%$ of ICU patients reporting dreams from their ICU admission was significant. Targeting long-term ICU patients with information and comprehensive discussion about different scenarios of ICU experiences may assist them in regaining a fulfilling life after discharge from ICU.

\section{COMPARISON OF FACTUAL MEMORIES OF ICU EX- PERIENCES AND DELIRIUM STATUS TWO YEARS AFTER DISCHARGE}

BL Roberts ${ }^{1}$, CM Rickard ${ }^{2}$, D Rajbhandari ${ }^{3}$, P Reynolds $^{2}$

1 Sir Charles Gairdner Hospital, Perth, Western Australia, Australia;2 Monash University, Latrobe Regional Hospital, Traralgon, Victoria, Australia;3 Royal Prince Alfred Hospital, Sydney, New South Wales, Australia

\section{Background}

Patients who require Intensive Care Unit (ICU) treatment may encounter several untoward experiences lasting well beyond the physical recovery of their illness. These can include factual memories of ICU and may be perceived as stressors by the patient. The presence of several such stressors can lead to the development of reduced quality of life and/or post-traumatic-stress-disorder (PTSD).

\section{Objectives}

To describe the relationship between patients' observed behavior using a validated screening tool during their ICU admission, their recall of factual memories from ICU and subsequent psychological outcome.

\section{Methods}

Following institutional ethics approval we conducted a prospective cohort study using the interview technique. The cohort was assembled from 152 patients who participated in a previously conducted multi-centre study of delirium incidence in Australian ICUs. The interviews involved a mixture of closed- and open-ended questions. Qualitative responses regarding factual memories were analyzed using thematic analysis. A 5-point Likert scale with answers from "always" to "never" was used to ask about current experiences of dream, anxiety, sleep problems, fears, irritability and / or mood swings. Scoring ranged from 6 to 30 with a midpoint value of 18 indicating a threshold value for diagnosis of PTSD.

A p-value of $<0.05$ was considered significant for all analyses.

\section{Results}

Forty-one patients $(40 \%)$ consented to take part in the follow-up interview out of 103 potential participants. 18 patients (44\%) had been delirious and 23 patients (56\%) non-delirious during the ICU admission. The non-participants $(n=62)$ formed a control group to ensure a representative sample. $83 \%(n=34)$ reported factual memories either with or without recall of dreaming. Factual memories were significantly less common (66\% c.f. $96 \%$ ) in delirious patients (OR 0.09, 95\% Cl 0.01-0.85, p=0.035). Five topics emerged from the thematic analysis: "procedures" i.e. endo-tracheal tube, "staff" i.e. nursing and medical staff, "comfort" (thirst or feeling secure), "visitors", and "events" ie. waking up. On current experiences five patients $(12 \%$ ) (four non-delirious and one delirious) scored $>18$ indicative of symptoms of PTSD. This did not reach a statistically significant difference. Memory of transfer out of ICU was less frequent amongst delirious patients $56 \%(n=10)$ than non-delirious $87 \%(n=20)(p=0.036)$.

\section{Conclusions}

Most patients had factual memories of their ICU stay. However, delirious patients had significantly less factual recall than non-delirious. Adverse psychological sequelae expressed as PTSD was uncommon in our study.

Every attempt must be made to ensure the ICU environment is as hospitable as possible to decrease the stress of critical illness. PostICU follow-up should include filling in the "missing gaps" and ongoing explanations in a caring environment may assist the patient in making a complete recovery both physically and mentally.

\section{RECORDS IN-HOSPITAL CARDIOPULMONARY RE- SUSCITATION: VALIDATE AND APPLICABILITY OF AN INSTRUMENT}

AP Boaventura, IEM Araújo

Universidade Estadual de Campinas - UNICAMP

\section{Background}

Usually, during in-hospital cardiopulmonary resuscitation (CPR), there is lack of comprehensive records about the whole procedure, or, more often, they are incomplete, reporting only the times of cardiac arrest (CA) and death. OBJECTIVES: The objectives of the present study were to validate and to apply an instrument elaborated to record in-hospital CPR maneuvers in a teaching institution. Validation is fundamental before using an instrument, it verifies the quality of the application of an instrument in a determined population, it is also going to perceive how the instrument behaves in the environment in which is implemented, to do that, the instrument was submitted to the validation of content and its applicability was also verified.

\section{Methods}

The instrument was previously validated by experts judges, and then it was applied by registered nurses at the emergency room, 


\section{Nursing Abstracts from the 2nd Conference of the World Federation of Critical Care Nurses:}

adult intensive care unit, clinical and surgical emergency wards, during cardiac arrest events in order to evaluate its practical applicability.

\section{Results}

Data analysis showed that there was no disagreement among judges regarding the instrument's intelligibility $(p=0,353)$, objectivity $(p=0,333)$ and organization $(p=0,107)$. (Cochran's test). Fiftyfour records of in-hospital CPR have been done, and the nurses were trained to evaluate the instrument's utility, practicability and objectivity, with more than $90 \%$ of positive answers. Information concerning patient's identification $(92,7 \%)$, cardiac arrest characterization $(72,2 \%)$, CPR maneuvers $(81,8 \%)$, post-CPR procedures $(89,6 \%)$, team of attendance $(27,4 \%)$ and general annotations $(7,4 \%)$, were possible to be retrieved from the applied instrument.

\section{Conclusions}

It was concluded that an adapted instrument for recording inhospital CPR procedures could be validated and easily applied by nurses in a teaching hospital. The utilization of this instrument would enable to stimulate to practical of records, news training, direct investments and adequate in-hospital CPR maneuvers in the units for critical ill patients and contribute to the improvement during in-hospital cardiopulmonary resuscitation.

0157 MEANINGS AND ATTITUDES: IS THERE ANY CORRELATION BETWEEN THESE TWO PSYCHOSOCIAL VARIABLES IN CARDIAC SURGERY PATIENTS?

AF Miranda1, MCBJ Gallani², S Araújo ${ }^{2}$

1 State University of Campinas/ Paulista University-Campinas-SPBrazil;2 State University of Campinas-Campinas-SP-Brazil

\section{Background}

Meaning occurs in a representational level where the stimulus that comes from the environment is projected in the brain, and at this point, an inside answer leads to an internal response (the meaning), which is a reaction associated to what a person has learnt between the answer to a concrete object and the sign. To sum up, the sign is something that is accepted to represent something else. The concept of attitude has been used to understand and to explain social behavior since 1918. Many definitions of attitude were presented by several authors since then. However, after 1950s, it was adopted a definition that is almost worldwide accepted, that considers many components. So the attitude was defined as "a complex system, including the people's beliefs about the object, their feelings to the object and the tendency of their actions related to the object".

\section{Objectives}

This study aims to verify the correlation among patients' meanings and attitudes related to cardiac surgery.

\section{Methods}

First of all two instruments of measurement were developed and validated: one to identify the patients' meanings related to cardiac surgery - Meanings of Patients related to Cardiac Surgery (SPCC), and the other instrument was developed to identify the patients attitudes related to cardiac surgery - Beliefs, Values and Feelings (CVSCC). The SPCC scale was composed of three factors called: Factor $\mathrm{A}=$ Concepts of positive meanings; Factor $\mathrm{B}=$ Concepts of negative meanings; Factor $\mathrm{C}=$ Concepts of ambiguous meanings. The CVSCC scale was composed of six factors called: Factor
$1=$ Negative feelings about cardiac surgery; Factor $2=$ Beliefs about benefits gained throughout a cardiac surgery; Factor $3=$ Trust on health staff and also on the cardiac surgery; Factor $4=$ Hope/faith in succeeding in cardiac surgery; Factor $5=$ Positive feeling that the surgery is necessary; Factor $6=$ Uncertainty about the result of cardiac surgery. A hundred twenty five patients in cardiac surgery preoperative period were interviewed. The data was analyzed by Pearson's correlation coefficient.

\section{Results}

There were some correlations between the two scales, but all of them showed a weak magnitude of signification. Concepts of positive meanings had a positive correlation with hope/faith in succeeding in cardiac surgery. There was a negative correlation between concepts of negative meanings and trust on health staff and also in the cardiac surgery. It also had a positive correlation among the concepts of ambiguous meanings and negative feelings about cardiac surgery, and positive ones that the surgery is necessary and uncertainty about the result of cardiac surgery.

\section{Conclusions}

Despite the fact that the correlations between the two scales had been weak and the statistics analysis didn't allow us to differentiate which variable was the cause or effect, the hypothesis that the meanings of patients about cardiac surgery have influence in their attitudes can be considered valid. This information, allow us to have a deeper view about the patients and it leads us to plan a more assertive nursing care.

\section{NURSE-DRIVEN INSULIN TITRATION IS EQUALLY EFFICACIOUS AND SAFE IN A CARDIOTHORACIC ICU}

PR Roberts, EH Kincaid, ND Kon, MK Williamson, MG Reichert, DL Bowton

Wake Forest University Baptist Medical Center

\section{Background}

In 2001, results of a large randomized ICU trial indicated that intensive glucose control decreased morbidity and mortality in a surgical ICU. In that trial blood glucose (BG) levels were maintained between $80-110 \mathrm{mg} / \mathrm{dL}$ by use of insulin infusions administered by protocol. In 2003, we began use of a similar detailed protocol order set to maintain glucose levels between $80-110 \mathrm{mg} / \mathrm{dL}$ in cardiothoracic (CT) surgery patients. However, despite various types of educational efforts we noted significant problems with nursing satisfaction in managing glucose in this manner.

\section{Objectives}

We sought a system that would achieve glycaemic control that would have improved nursing satisfaction and "buy-in." Here we report the results of this experience.

\section{Methods}

During June through September 2004, 149 patients were treated with our insulin infusion titration order set by the bedside nurse based on frequent BG measurements. Then during October 2004 through January 2005, 150 patients were treated via insulin infusions with titrations made by the bedside nurse based on her/his clinical judgment using similar frequent BG monitoring. In both study periods the glucose goal was $80-110 \mathrm{mg} / \mathrm{dL}$. Data collected included all glucometer readings (Lifescan, Johnson \& Johnson, USA) obtained during the ICU stay. Descriptive statistics of glucose control and hypoglycemia are presented. 


\section{Results}

\begin{tabular}{|l|l|l|}
\hline Treatment Group & $\begin{array}{l}\text { Detailed Order Set } \\
\text { Driven Titration }\end{array}$ & $\begin{array}{l}\text { Nurse-Driven } \\
\text { Titration }\end{array}$ \\
\hline Number of Patients & 149 & 150 \\
\hline \# BGs Performed & 10,044 & 7,249 \\
\hline Mean BG \pm SEM $(\mathrm{mg} / \mathrm{dL})$ & $118.0 \pm 0.4$ & $117.3 \pm 0.4$ \\
\hline$\%$ BG $80-110(\mathrm{mg} / \mathrm{dL})$ & 43.6 & 45.2 \\
\hline$\%$ BG $70-150(\mathrm{mg} / \mathrm{dL})$ & 81.8 & 85.9 \\
\hline$\%$ BG Below $40(\mathrm{mg} / \mathrm{dL})$ & 0.02 & 0.00 \\
\hline \#GG Below $40(\mathrm{mg} / \mathrm{dL})$ & $2^{*}$ & $0^{*}$ \\
\hline$\%$ Nurses Satisfied & 67 & 96 \\
\hline with Protocol & & \\
\hline
\end{tabular}

*No evidence of complications from hypoglycemia was documented.

\section{Conclusions}

We conclude that use of the 2 different systems led to clinically equivalent and reasonable glycemic control with the mean BGs of $117-118 \mathrm{mg} / \mathrm{dL}$, despite both regimens resulting in BGs slightly above the goal. Hypoglycemia was an uncommon event, and its incidence was similar between control systems. In our experience, it is difficult to maintain BG between 80 and 110 in the ICU setting. We believe ICU nurses can use their judgment to titrate insulin, as long as surveillance systems are in place to detect problems, monitor for the incidence of hypoglycemia, and provide feedback. However, the success of our nurses with this regimen may have been enhanced since they had been aggressively managing glucose for over a year with an order set. Intensive glucose control requires ongoing nursing efforts and their "buy-in" is important. Surveys of our nurses indicate significant improvements in their satisfaction using nurse-driven titration. Currently we are assessing whether nurse-driven insulin titration is safe and can achieve BG control throughout other ICUs in our hospital.

0174 THE MOST FREQUENT NURSING DIAGNOSIS IN THE PATIENTS SUBJECTED TO THE CONTINUOUS RENAL REPLACEMENT THERAPIES

RCS Souza, CS Matsuba, AL Cheregatti, AM Silva

Hospital do Coraçao-Associaçao do Sanatorio Sirio

\section{Background}

Cardiopathy patients develop in specific situations Acute Renal Insufficiency (IRA), which triggers important physiological alterations and require treatment, due to their haemodynamic repercussions. Some of the most used treatment methods are called the Continuous Renal Replacement Therapies (CRRT). The nursing team is responsible for the direct assistance to the patient, it is essential that the nurse takes appropriate decisions regarding the necessary interventions to obtain the expected results. Therefore, it is vital to identify the nursing diagnosis in this group of patients, this is an essential step in the systematization of the nursing assistance.

\section{Objectives}

Identify the most frequent nursing diagnosis in the patients subjected to Continuous Renal Replacement Therapies.

\section{Methods}

A descriptive and prospective study, that was held in the period between April and September 2004 in 12 patients subjected to CRRT. The data collection was done through an instrument specially formulated for the research.

\section{Results}

Eight (8) most common nursing diagnoses were identified according to the relative an absolute frequency of related factors present in such group of patients. They represent the physiological and/or pathological answers that those individuals present in this specific situation and contribute to direct the nursing actions.

\section{Conclusions}

This study made evident the importance of identifying the nursing diagnosis in a group of patients which go through specific situations to obtain quality nursing assistance, directed to the individual real needs. In doing so, it becomes possible for the nurse to propose actions that ensure a good effectiveness and efficiency based on scientific principles.

\section{THE USE OF RESPIRATORY PHYSIOLOGY KNOWL- EDGE IN CRITICAL CARE NURSES' CLINICAL DECISION- MAKING}

AM Pirret

Counties Manukau Health, Auckland, New Zealand

\section{Background}

The knowledge and experience of expert nurses has long been recognised as contributing to early identification of patient problems, early intervention and an improved patient outcome.

\section{Objectives}

The present study sought to answer the research question, following completion of a critical care specialty practice programme, do nurses use knowledge of respiratory physiology in their clinical decision-making?

\section{Methods}

An evaluation methodology using a managerial perspective was used to compare the use of knowledge of respiratory physiology in critical care nurses' clinical decision-making with the respiratory physiology recommended in the New Zealand Standards for Critical Care Nursing Education (Critical Care Nurses' Section, 2000). Prior to the implementation of this research, ethical approval was obtained from both the university and regional ethics committees, and written approval gained from the participants, critical care unit managers of each research site, the Regional Maori Research Review Committee and the Regional Development Office of the appropriate District Health Board. Confidentiality and anonymity of the participants and critical care units involved in this study were maintained throughout the research process. Using intensity sampling, 27 nurses who had completed a critical care specialty practice programme and who were currently working in the critical care units of two tertiary hospitals in a large metropolitan city within New Zealand were selected. Quantitative and qualitative methods were used to collect data. Data analysis was completed using descriptive statistics, correlations and identification of common terms and themes.

\section{Results}

The results showed that following completion of a critical care programme, critical care nurses demonstrated a low to medium level of knowledge of respiratory physiology in their clinical decision-making. In the total group, no statistically significant associations were found between the use of knowledge of respiratory physiology in clinical decision-making and age, experience, academic level, use of guidelines and protocols, standards and integrated care path- 
ways, conference attendance, reading of journals and accessing the World Wide Web for literature. Qualitative analysis identified factors contributing to the low to medium use of knowledge as being nurses' high reliance on intuitive knowledge, lack of in-depth discussion of respiratory concepts in critical care programmes, lack of opportunity in the clinical practice environment to discuss respiratory physiology and lack of collaborative practice.

\section{Conclusions}

The study identified the need for more collaborative practice and for clinical nurse educators firstly, to have a closer relationship with the critical care programme providers to ensure adequate theoretical content and secondly, to work with nurses in the clinical setting.

\section{Reference}

Critical Care Nurses' Section. (2000). New Zealand standards for critical care nursing (2nd ed.). Wellington: New Zealand Nurses' Organization.

\section{PERCEPTION OF PAIN IN ONCOLOGIC AND NO ONCOLOGIC CHILDREN. A NURSING VIEW}

FSV Tourinho-Pereira ${ }^{1}$, MC Azzi ${ }^{2}$, MFB Oliveira ${ }^{2}$, T Ruzza ${ }^{2}$, FA Pereira ${ }^{3}$

1 Methodist University of Piracicaba - São Paulo - Brazil;2 São Francisco University - Bragança Paulista - São Paulo -Brazil;3 MEDICAL Hospital - Limeira - São Paulo - Brazil

\section{Background}

In the last times, several advances in the treatment of the infantile cancer had occurred, and cure rates reached $70 \%$ of the cases. To evaluate the pain of these children, face diagrams are used among others instruments. However, one of the great imperfections in the treatment of pain is the lack of professional preparation. The idea of this work was born of the necessity in identifying how the nurse performance could be improved in the context of pain in pediatrics.

\section{Objectives}

The objective of this study was to identify the perception of pain of children with cancer and compare with children who do not have cancer and verify what is pain for them.

Methods: In this qualitative, descriptive study, three children treating cancer and 6 children without cancer had participated. The methodology used a theater, with the participation of the researchers and children as actors. These dressed up as forest animals according to the scene and questions had been carried through, making possible the understanding of pain.

\section{Results}

Characterizing and quantifying pain, it was possible to understand that pain for child exceeds the physicist, include the emotional and spiritual suffering, conducting itself for a series of feelings.

\section{Conclusions}

The nursing team must pay more attention in pain information provided by child and its family, learning to hear and believe more when child complains about pain. This attitude makes the professional capable to see pain as the children see, promoting an quality and humanity assistance, for the child and family, being possible to have cancer without having pain.

\section{ROUTINE REPLACEMENT OF INTRAVENOUS FLUID BAGS DOES NOT PREVENT MICROBIAL COLONIZATION: CONTROLLED COHORT STUDY}

CM Rickard ${ }^{1}$, B Vannapraseuth $^{2}$, LJ Keene ${ }^{3}$, MR Mcgrail ${ }^{1}$, S Rambaldo $^{2}$, CA Smith ${ }^{1}$

1 Monash University, Traralgon, VIC, Australia;2 Gippsland Pathology, Traralgon, VIC, Australia;3 Latrobe Regional Hospital, Traralgon, VIC, Australia

\section{Background}

Health Care Acquired Infection (HCAl) occurs in $6-12 \%$ of inpatients causing suffering, risk of death and costs [1]. Many HCAls occur in intravenous catheters. A historical preventative strategy is replacement of intravenous infusion systems at routine intervals. Research suggests tubing and burettes may be used for $\geq 7$ days, however, the effect of fluid bag use duration is unknown, and daily replacement continues in many hospitals. [2] The CDC Guidelines state ecommendations for length of use cannot be given due to lack of evidence.[3]

\section{Objectives}

Determine intravenous fluid bag microbial colonisation rates after $\geq 24$ hours use. Document current clinical length of use for fluid bags. Investigate any time-dependant relationship to colonisation. Develop recommendations for the duration of intravenous fluid bag use.

\section{Methods}

Controlled cohort study in a 10-bed critical care unit and 257-bed regional referral hospital. After ethical approval, samples were collected using aseptic technique from 264 fluid bags used $\geq 24$ hours with peripheral, central venous or peripheral arterial lines. A control group of 261 never-opened fluid bags were also sampled. Fluids included saline and dextrose based crystalloids. Samples were cultured for 48 hours on blood; microbiological colony counts and speciation were recorded. Laboratory staff were blinded. Data on potential risk factors was recorded including patient age, use of a burette, fluid type and intermittent disconnection of the intravenous tubing.

\section{Results}

Patient-related fluid bags were used for a median of 34 hours (SD 1.8, range 21-185 hours/1-7 days). Colonisation occurred in patient-related samples $(2.7 \%)$ and controls $(6.9 \%)(p=0.02)$. Organisms in both groups were mainly dnase and coagulase negative staphylococcus at low counts. Median duration of bag use was not different ( $p=0.99)$ between colonised (35.0 hours) and sterile patient-related samples (34.0 hours).

\section{Conclusions}

Colonised samples in both groups likely represent sampling contamination rather than fluid bag colonisation. Our results suggest intravenous fluid bags are not colonised after 24 hours of clinical use and there is no benefit in routine replacement. The risk of contamination when disconnecting the bag for routine replacement may be higher than the chance of removing a colonised bag. Approximately 500 million intravenous catheters are used annually. The results have implications for infection control, nursing time, equipment costs and environmental waste. 


\section{References}

1. Plowman R. The socioeconomic burden of hospital acquired infection. Eurosurveillance 2000; 5:49-50.

2. Rickard CM, Lipman J, Courtney M, Siversen R, and Daley P. Routine changing of intravenous administration sets does not reduce colonization or infection in central venous catheters. Infect Control Hosp Epidemiol 2004; 25:650-655.

3. O'Grady NP, Alexander M, Dellinger EP, et al, Guidelines for the prevention of intravascular catheter-related infections. Am J Infect Control 2002; 30:476-489.

0193 THE EFFECTIVENESS OF AN INTERVENTION TO INCREASE PUBLICATION RATES BY HEALTH PROFESSIONAL ACADEMICS: RESULTS OF PROSPECTIVE COHORT STUDY

CM Rickard, CR Jones, MR Mcgrail, A Robinson, M Burley

Monash University, Traralgon, VIC, AUSTRALIA

\section{Background}

Health professionals are under increasing pressure to publish in the refereed literature. Despite this, many do not publish, and much research goes unreported.

\section{Objectives}

To evaluate the effectiveness of an intervention to increase publication rates of health professionals

\begin{abstract}
Methods
Participants were academic health professionals from fields including intensive care, emergency and nursing. An anonymous, self-report, web-based survey with a mix of short-answer and open-ended questions was used. 10 attendees at a one-week consultant-led writing course, followed by ongoing monthly peer-support meetings were invited to participate. 2 years of data pre and post the writing course was obtained on publication rates and types, reasons for attendance and best and worst aspects. Quantitative and qualitative analysis occurred.
\end{abstract}

\section{Results}

Most participants had no publication experience before the writing course. Afterwards, most published at least once. Overall publication rates increased four-fold. This resulted in average publication rates per person of 2 refereed articles per year as first or co-author. Other reported benefits were support and motivation from the peer-group, team-building, increased collaborative writing and researching, plus improved confidence in writing ability. Excessive workloads were the most highly cited impeding factor.

\section{Conclusions}

Writing for publication is a skill that can be learned. The evaluated model of a formal writing course, followed by informal monthly meetings, is an effective way of increasing publication rates.

0225 COORDINATION AND DONATION OF "INTRA AND EXTRA-HOSPITAL" CADAVER DONORS. "THE PAMPLONA MODEL". SEQUENCE OF TASKS PERFORMED 1992 2004

E Maraví-Poma 1 , A Maraví-Aznar ${ }^{1}$, R Teijeira ${ }^{2}$, A Hidalgo ${ }^{2}$, A Martín ${ }^{1}$, Team Sos-Navarra3

1 ICU \& Transplant Coordination Team Virgen del Camino Hos- pital. Pamplona; 2 Navarra Institute of Legal Medicine;3 SOS-Navarra 112

\section{Objectives}

Sequence of tasks performed in a model for detecting and extracting extra-hospital (EH) cadaver donor tissue from cardio-respiratory arrest (CRA) and Intra-hospital (IH) from cerebral death (CD), at a non-neuro-traumatolological ICU.

\section{Methods}

Multi-center and prospective study from 1992-2004, centralized in the Virgen del Camino Hospital ( $\mathrm{VCH})$.

\section{Results}

In 1990 a transplant team composed of an intensive medicine physician and two nurses was formed. A potential tissue donor is any cadaver from 1 to 60 years old which meets general donor criteria. The majority result from traffic accidents. The sequence of the tasks: 1. Alert from SOS-Navarra (Central Assistance Service, public). $\mathrm{VCH}$ transplant Coordinator is advised of the existence of a cadaver. The alerts come from different locations and hospitals where fatal accidents occur. The forensic physician on duty asks the examining magistrate for legal permission. Criteria for a potential tissue donor are verified. This phase lasts between $30^{\prime}$ and two hours. 2. Criteria for selecting, searching and contacting patients' relatives. Application of the different protocols for transporting, evaluating, and selecting the cadaver and enabling the forensic physician to act. The deceased's relatives are then located to obtain family permission, if possible in writing or by means of a telephone call. Blood samples are taken and data for the clinical history is gathered. By the time this phase is completed, between 3:30 and 12 hours have elapsed. 3. Serology, extraction teams and transportation are notified. When the cadavers meet the criteria they become real donors. Firstly, the cornea extraction team intervenes in the autopsy room of the Navarra Institute of Legal Medicine or the $\mathrm{VCH} /$ Hospital surgery room. Cardiac valves, large vessels and bone tissue are then extracted in the Surgery room of the University of Navarra Clinic (UNC). Following local decontamination, in accordance with approved protocols, corneas, or vascular/bone tissue are prepared and stored in tissue banks at the VCH or CUN. 4. Conservation, storage, distribution and transportation of tissue. 5 . Distribution of previously agreed incentives. The Coordinator informs the Judge and the Autonomous Region Coordination. The incentive distribution is drawn up for medical professionals involved in the detection, selection, serology, extraction and transportation, excluding the transplant activity. The coordination team is available 24 hours/day and 365 days/year. The work schedule is as follows: $8: 00-15: 00$ hours $=26 \%$ of the time; from 15:00 $-20: 00=$ $25 \%$; from $20: 00-24: 00=30 \% \&$ from $00: 00$ to $8: 00=19 \%$.

\section{Conclusions}

The Pamplona Model has made important organizational progress and it is the first to be implemented at a Hospital ICU which does not have it own organ donors. Detection is centralized in a public service (SOS-Navarra) which ensures accessible and economical coverage in the entire area.

O227 EARLY IDENTIFICATION AND PREVENTION OF ICU DELIRIUM: AN EVIDENCE BASED PRACTICE APPROACH EM Santiago, R Newhouse

Johns Hopkins Hospital, Baltimore, Maryland, United States of America 


\section{* Nursing Abstracts from the 2nd Conference of the World Federation of Critical Care Nurses:}

\section{Background}

Delirium in the Intensive Care Unit (ICU) has been identified in the literature as an independent predictor of patient morbidity and mortality. The occurrence of ICU delirium has been linked to poor patient outcomes, increases in ICU length of stay, and hospital costs. In addition, evidence links the incidence of delirium during a hospital stay with persistent cognitive disability. Despite practitioner awareness of the high incidence of ICU delirium, there is a general disconnect between perceived importance, early identification, and intervention on modifiable risk factors that could prevent its occurrence. In ICU's, delirium is frequently under recognized, under diagnosed, and mismanaged. This may be due in part to the level of nurse knowledge of modifiable risk factors. Recent evidence identifies the elderly population to be at an increased risk of developing ICU delirium. Patients 65 years old and older account for more than $60 \%$ of all ICU days. Research on hospitalized elderly patients has identified predisposing and precipitating factors for the development of delirium. In addition, it has been demonstrated that practical interventions targeted towards recognizing these risk factors and implement proactive strategies are effective in preventing delirium.

\section{Methods}

Throughout the months of March until June of 2005, an evidence based practice project will be conducted at the Johns Hopkins Hospital Surgical Intensive Care Unit. Using The Johns Hopkins Nursing Evidence Based Practice Model (Newhouse et. al., 2005) a multidisciplinary team of nurses and physicians will conduct a literature review on the identification of modifiable risk factors and early prevention of ICU delirium.

\section{Results}

Through this evidence-based practice project, we will develop clinical practice guidelines on the identification of modifiable risk factors and nursing interventions to prevent ICU delirium. The Johns Hopkins Nursing Evidence-based Practice model will be used to translate research findings into scientifically sound nursing clinical practice.

\section{Reference}

Newhouse, R., et al. (2005). Evidence-based practice: a practical approach to implementation. Journal of Nursing Administration, 35(1), pp.35-40.

\section{MONITORING HUMIDIFICATION OF VENTILATED PATIENTS \\ E Gonzalez, X Arrieta, M Suarez \\ Clinica Las Condes}

\section{Background}

Keeping an adequate humidity of the inspired air in patients exposed to mechanical ventilation becomes important to avoid the formation of mucus plugs and bronchial inundation or changes of the hydric balance. We found in our clinical work that the always quick and timely response of the nursing team to hemodynamic and respiratory alarms were not that efficient compared to the alarms of humidifier of the mechanical ventilators (MV).

\section{Objectives}

To evaluate the response of the nursing team to humidifier alarms of $\mathrm{MV}$ and observe their attitude with respect to possible failures such as temperature, a smaller size of the sensor cable than the circuit, or wrong calibration of the temperature of the chambers that induce an increase or decrease of the temperature of patients airway.

\section{Methods}

Non programmed supervision was conducted to prevent personnel from knowing that the study was being conducted. An analysis worksheet to patients in mechanical ventilation admitted to the Pediatric Intensive Care Unit in June and July 2004.

\section{Results}

Seventy-eight non programmed supervisions were done finding 34 episodes of malfunction of the humidifiers.

\begin{tabular}{|l|l|l|l|l|}
\hline $\begin{array}{l}\text { Type of inadequate } \\
\text { function }\end{array}$ & $\begin{array}{l}\text { Corrects } \\
\text { Failure }\end{array}$ & $\begin{array}{l}\text { Silences } \\
\text { Alarm }\end{array}$ & $\begin{array}{l}\text { No } \\
\text { Response }\end{array}$ & $\mathbf{n}$ \\
\hline Triggered Alarm & 20 & 5 & 3 & 28 \\
\hline Humidifier Off & 3 & 0 & 0 & 3 \\
\hline No Water in Humidifier & 3 & 0 & 0 & 3 \\
\hline
\end{tabular}

\section{Conclusions}

A failure in the humidifier was found in $43.5 \%$ of the supervision. In $8(23.5 \%)$ of them, there was no response of the nursing team to correct the malfunction detected. This could cause a deterioration in the critical condition of our patients. This result motivated a programmed intervention to change these results. A lecture type class was given to the nursing personnel of the whole institution. It is very important that every nursing action should be supervised regardless of how simple it may seem. The successful care of a critical patient depends on paying continuous attention to details.

\section{PEDIATRIC HOST DEFENSE: NOSOCOMIAL INFEC- TION, SEPSIS AND NEW TREATMENT MODALITIES}

TL Haugen

BC Children's Hospital, Vancouver, BC Canada

\section{Background}

The pediatric immune system is less efficient than that of the adult. When combined with an invasive environment, such as the pediatric intensive care unit (PICU), many health care challenges arise. Primary bacteraemia is the major nosocomial infection (NI) in neonatal and pediatric populations (Richards, Edwards, Culver, Gaynes \& the Nosocomial Infections Surveillance System, 1999) and substantially increases their risk of developing severe sepsis. Severe sepsis gives rise to an average annual mortality rate in the United States of $30 \%$ in adults and $10 \%$ in children, accounting for approximately 215,000 deaths and hospital expenditures of over 16 billion dollars (Angus et al., 2001). In light of these statistics, innovative approaches for treating sepsis are being investigated.

\section{Objectives}

Overview: Sepsis and sepsis related sequelae; Immune system: 1st, 2nd and 3rd lines of defense.

Discuss: Pediatric NI within the PICU environment; Effects of Transient Immunocompromise (TI) in critically ill patients; How TI and $\mathrm{NI}$ interrelate with breached barriers to increase severe sepsis development; Limitations and new directions of conventional sepsis management; Emerging therapeutic modalities that focus on molecular facets of the hematological system to decrease sepsis related mortality; Coagulation and inflammatory response changes characteristic of the sepsis process. 


\begin{abstract}
Methods
Literature Search: NI, Immune system: differences between neonates, children and adults and effects of the critical care environment on the immune system; Severe Sepsis: Mortality Incidence, coagulation and inflammatory changes, conventional management, clinical trials of innovative therapies (neonate, pediatric, adult).

\section{Results}

Of the Phase III Clinical Trials, only drotrecogin- (activated) has been approved by authorities of the U.S. Federal Drug Administration (FDA) (FDA News, 2001) and the Biologics and Genetic Therapies Directorate (BGTD) of Health Canada (Canada news wire, 2003), for use in adults with severe sepsis. However, a worldwide trial is currently underway to assess drotrecogin- (activated) benefits in the pediatric population. Additionally, use of CSFs to halt septic related mortality in septic pre-term neonates, are being investigated further in a large phase III trial in the UK (National Perinatal Epidemiology Unit, 2004).
\end{abstract}

\section{Conclusions}

Severe sepsis, involving altered coagulation and inflammatory defenses, is on the rise consuming thousands of lives and billions of health care dollars per annum in the U.S. alone. The intensive care environment houses severely ill, immuno-compromised patients that when combined with a highly invasive environment can increase susceptibility to $\mathrm{NI}$ and the development of severe sepsis. The use and continued research of molecular based treatment strategies, combined with improvements in conventional sepsis management, and reduction of $\mathrm{NI}$ through bedside practice changes, will impact and improve overall mortality related to the vast and still somewhat un-chartered process of severe sepsis.

\section{SEDATION AS A COMPONENT OF THE CRITERIA TO PERFORM A SPONTANEOUS BREATHING TRIAL}

S Arias-Rivera, MM Sánchez-Sánchez, R Santos-Díaz, MJ GallardoMurillo, R Sánchez-Izquierdo, F Frutos-Vivar

Intensive Care Unit, Hospital Universitario de Getafe

\section{Objectives}

To determine the importance of sedation as a component of the criteria to perform a spontaneous breathing trial in mechanically ventilated patients.

\section{Methods}

Prospective study in a medical-surgical ICU of 18 beds. We included patients with mechanical ventilation longer than 48 hours who fulfilled criteria of weaning and were extubated. Demographic data, doses of sedatives, daily level of sedation, date of start mechanical ventilation, date of clinical criteria to spontaneous breathing trial, date of performance the spontaneous breathing trial, date of extubation and date of reintubation were registered. Clinical criteria of weaning were: a) Improvement or resolution of the reason for mechanical ventilation, b) $\mathrm{PaO} 2 / \mathrm{FiO} 2>200$, c) Absence of vasoactive agents, d) Core temperature $<38$ oC, d) Hemoglobin > 8gr/dl.

\section{Results}

We included 171 patients that were extubated after a successful spontaneous breathing trial: 48 patients $(28 \%)$ in the first attempt of spontaneous breathing trial and $123(72 \%)$ in successive daily trials. The patients who failed in the first spontaneous breathing trial were more sedated on the day of the test than the patients who successfully passed the trial: mean in the Glasgow-Cook scale [11(4) vs. $14(3) ; p<0.05]$. We do not observed significant differences in the level of sedation either day of the extubation or in the rate of reintubation (15\% vs 19\%) between the patients extubated in the first spontaneous breathing trial and the patients who failed .The median of the stay in the intensive care unit of the successfully extubated patients in the first spontaneous breathing trial was lower [7(5.14) vs 13(7.21); $\mathrm{p}<0.05]$.

\section{Conclusions}

We have found that in our cohort of patients, the level of sedation is a factor to evaluate in patients who fulfilled criteria to a spontaneous breathing trial.

\section{LEVEL OF SEDATION DURING THE MECHANICAL VENTILATION: COMPARISON BETWEEN MIDAZOLAM AND PROPOFOL}

S Arias-Rivera, R Sánchez-Izquierdo, MM Sánchez-Sánchez, R Santos-Díaz, MJ Gallardo-Murillo, F Frutos-Vivar Intensive Care Unit, Hospital Universitario de Getafe

\section{Objectives}

To determine the level of sedation of mechanically ventilated patients over the course of mechanical ventilation and weaning.

\section{Methods}

Observational and prospective study performed in a medical-surgical of 18 beds during 18 months. The level of sedation was determined by Glasgow sedation scale modified by Cook (G-C) that ranges from 0 to 15 points. Three levels of sedation were defined: a) High sedation: patients with GC ?7 points, b) Optimal sedation: patients with GC 8-12 points, c) Low sedation: patients with GC ?13 points. We evaluated the level of sedation during two periods: a) From the patient was intubated to the first spontaneous breathing trial was carried out, b) From the first spontaneous breathing trial to extubation. Daily dose of midazolam, propofol and morphine was registered.

\section{Results}

We included to 176 consecutive mechanically ventilated patients longer than 48 hours. We observed that the patients sedated with midazolam had a higher sedation, before spontaneous breathing trial than patients sedated with propofol $(p<0.05)$. Patients in which propofol was administered had a lower duration of weaning than patients sedated with midazolam (median: 1 day vs. 3 days; $\mathrm{p}<0.05)$.

\section{Conclusions}

In our cohort, we found that the sedation with midazolam produced a higher sedation during the time of mechanical ventilation and this is associated with a longer duration of the weaning compared with the sedation with propofol.

\section{INFLUENCE OF THE SEDO-ANALGESIA ON WEAN- ING FAILURE}

S Arias-Rivera, R Santos-Díaz, MJ Gallardo-Murillo, R Sánchez-lzquierdo, MM Sánchez-Sánchez, F Frutos-Vivar

Intensive Care Unit, Hospital Universitario de Getafe

\section{Objectives}

To determine if the doses of sedatives and analgesics administered during the mechanical ventilation are related with weaning failure 


\section{* Nursing Abstracts from the 2nd Conference of the World Federation of Critical Care Nurses:}

defined as failure of spontaneous breathing trial in two consecutive days or reintubation within 48 hours after extubation in patients who successfully passed the first spontaneous breathing trial.

\section{Methods}

Observational and prospective study in a medica-surgical ICU of 18 beds during 18 months. We included to 176 consecutive mechanically ventilated patients longer than 48 hours. Demographic data, mechanical ventilation data (period of intubation, weaning and reintubation), doses of sedatives (midazolam and propofol) and analgesics (morphine) were registered.

\section{Results}

Ninety-six patients (55\%) had a successful weaning and $80(45 \%)$ were failed ( 66 patients failed in two consecutive daily spontaneous breathing trial and 14 patients were reintubated). There were not differences in the median doses of sedatives and analgesics during the mechanical ventilation. The morphine administration in the previous 24 hours to spontaneous breathing trial was related to the successful test $[\mathrm{OR}=2.2(\mathrm{IC} 95 \%: 1,1-3)]$. Among the patients who received morphine we found differences in the dose received among those who had a successful extubación and those who failed (38 mgr/24h vs. $21 \mathrm{mgr} / 24 \mathrm{~h} ; \mathrm{p}<0.05$ ). We did not find differences in the level of conscience between both groups.

\section{Conclusions}

We have observed that success in the spontaneous breathing trial was related to the administration of a low dose of morphine (around $1.5 \mathrm{mg} / \mathrm{h}$ ) in the previous 24 hours.

\section{ICUCONNECT: COMMUNICATION BETWEEN IN- TENSIVE CARE CLINICIANS - IT'S ONLY A KEYSTROKE AWAY!}

DC Kowal, K Rolls, AR Burrell

Intensive Care Coordination And Monitoring Unit

\section{Background}

New South Wales, Australia, has 41 public intensive care units. 21 are located in the Sydney metropolitan area, 4 in the Greater Metropolitan area of Sydney, and 16 in the rural area. In units outside the metropolitan area, geographical distance from larger tertiary centres fosters a perception of professional isolation that may potentially impact on quality health care delivery to the critically ill patient. A statewide email list-server, ICUConnect was created in December 2003 to establish a communication network for ICU managers and educators, promoting ICU service delivery partnerships. The challenge was reciprocity, transforming members seeking information into members providing information. This peer-supported network granted participants the opportunity to be involved in a larger, statewide picture.

\begin{abstract}
Methods
Initially, nurse managers and clinical nurse consultants were entered onto the list-server; however during 2004 the demand for membership expanded to include clinical nurses, academics, allied health personnel, doctors, and staff from other critical care areas. A database facilitates analysis of ICUConnect usage. In May 2004, the first Annual User Survey, comprising a series of closed questions was conducted.
\end{abstract}

\section{Results}

From December 2003 to May 2004, ICUConnect membership grew from 130 to 191; comprising nurse managers or educators $(73 \%)$, clinical nurses $(6 \%)$, nurse research officers or equipment officers $(8 \%)$, medical officers $(9 \%)$ and allied health or academics (5\%). $46 \%$ of membership came from tertiary hospitals in the Sydney metropolitan area, $27 \%$ of members worked in metropolitan or outer-metropolitan centres and $24 \%$ of members came from the rural sector. Response rate to the survey, conducted via ICUConnect, was $27 \%$ (n51/191). $100 \%$ of respondents agreed that ICUConnect was beneficial to their ICU practice. $61 \%$ of respondents had made a new communication link with an ICU clinician outside their local area health service. Survey results highlighted that ICUConnect had addressed rural staff professional isolation with rural units contributing four times the number of queries as compared to metropolitan staff and metropolitan staff contributing five times the number of responses as compared with rural staff. Review of ICUConnect emails highlighted that activity focused on sharing policies and procedures relating to: ventilation, drug administration, intravenous access, infection control, and renal support therapies. Workforce and patient care issues also dominated on-line activity.

\section{Conclusions}

ICUConnect has proven to be a valuable tool for intensive care clinicians within New South Wales, demonstrating that ICU planners can be responsive to the needs of their stakeholders. Despite large geographical distances, well-established peer-supported networks lessen the sense of professional isolation. With a current membership of over 400, ICUConnect continues to infiltrate intensive care services and divisions, not only in New South Wales but also around Australia.

\section{A CHANGE MANAGEMENT PROJECT TO IMPROVE BREASTFEEDING INITIATION WITHIN A PEDIATRIC IN- TENSIVE CARE UNIT USING AN ACTION RESEARCH AP- PROACH}

GM Thompson

Our Lady's Hospital For Sick Children, Dublin, Ireland

\section{Background}

This project was aimed at improving breastfeeding initiation within an Irish paediatric intensive care unit. There was a cohort of infants in this unit not receiving any breast milk. Ireland's breastfeeding initiation rate of $36.9 \%$ is the lowest in Europe. A collaborative interdisciplinary team identified possible actions to improve breastfeeding facilitation within this environment.

\section{Objectives}

To improve breastfeeding initiation by a collaborative planned change, to increase staff awareness of the importance of breast milk for intensive care patients, and by ensuring that parents of newborn infants receive accurate, consistent and timely information.

\section{Methods}

An action research approach was used as this is specifically designed to bridge the gap between theory, research and realities of practice. A checklist to guide nursing practice was developed and introduced to the admission process, documenting that breastfeeding has been discussed and that written information has been given by the admitting nurse. The context of the change within this organization is described and change issues are identified. The literature pertaining to breastfeeding, change management and action research are reviewed. The driving and restraining forces and how this change was implemented in practice are outlined. The difficul- 
ties encountered and the complexities of the underlying issues are addressed.

\section{Results}

Audits of admission group both prior to and post introduction of the checklist showed an improvement from $17 \%$ of admissions receiving any breast milk to $56 \%$. The number of infants continuing to receive breast milk on discharge has risen from less than $10 \%$ to $43 \%$. The quantitative data arising from the audit process is presented graphically.

\section{Conclusions}

An interdisciplinary collaborative process identified many factors, which impede breastfeeding in this context concurring with the literature. A change to nursing practice was introduced which was within the autonomy of the change agent and time frame available. It achieved the planned objectives, introducing a process ensuring parents receive breastfeeding information. Thus giving them opportunity to make an informed feeding decision, based on their new and often unexpected crisis situation. Secondly, raising staff awareness as to the importance of breastfeeding in this environment. Change agents need to consider the context and culture of an organisation before implementing change, as issues in practice do not occur in isolation. Change is more likely to be acceptable if those affected participate in planning for it. The project evaluation undertaken to date suggests that, in spite of some problems with compliance this change has made a positive impact on practice. There are more babies receiving breast milk and more babies still breastfeeding on discharge. The paediatric intensive care nurse has a unique opportunity for educating parents about the health benefits of breast milk for their sick baby.

\section{CONTINUOUS HEMOFILTRATION IN PEDIATRIC CRITICAL CARE PATIENTS}

A Del Rio, B Fernandez, J Llosa, I Fuentes, A Donoso

Area de Cuidados Criticos Hospital Padre Hurtado, Santiago, Chile

\section{Background}

Continuous haemofiltration (CHF) is an essential procedure in critical care. However, application of this therapy to pediatric patients is associated with several problems due from their smaller body size and weight compared with adults.

\section{Objectives}

Review our experience in this procedure in the last five years in our PICU. Assess clinical efficacy and safety.

\section{Methods}

Reviewing patient's clinical records (1999-2004).

\section{Results}

28 patients treated with $\mathrm{CHF}, 50 \%$ males. Their body weight ranged from $4 \mathrm{~kg}$ to $59 \mathrm{~kg}$. $50 \%$ under one year old. The underlying disease was septic shock (57\%) and the clinical indication was renal failure (43\%). The mean CHF duration was 4.5 days. Blood access was provided in a veno-venous mode in all patients, femoral vein (54\%) and jugular-femoral (28\%). 9/28 patients (32\%) in continuous haemodiafiltration (CHDF). In 12/28 patients the CHF duration was $>3$ days. Regional anticoagulation was used in $7 / 28$ (25\%). The mean life time haemofilter used was 36 hours. The most frequent complications were circuit dysfunction (75\%) and tem- perature control of the patient $(50 \%)$. Of the 28 patients receiving CHDF, 16 patients survived without serious complications, achieving a survival rate of $57 \%$.

\section{Conclusions}

In pediatric critical care, CHF is safely applicable to the critically ill and it's a feasible therapy in a critical pediatric patient, without major problems or important complications, which allows us to improve the clinical management of our patients.

\section{NURSING CARE NEEDS AND THERAPEUTIC INTER- VENTIONS IN INTENSIVE CARE UNITS: A COMPARATIVE STUDY OF ELDERLY AND NON-ELDERLY PATIENTS}

KG Padilha1, JT Ciampone1, LA Gonçalves2, FOM Maia2

1 University of São Paulo - School of Nursing;2 Hospital Universitário da Universidade de São Paulo

\section{Objectives}

The objectives of this study were to compare the nursing care needs and the therapeutic interventions carried out on elderly and nonelderly patients in ICU, according to the Nursing Activities Score (NAS).

\section{Methods}

The sample was composed of 50 adult patients admitted into the ICU of a university hospital in the municipality of São Paulo, with a starting date of September 26, 2003. The NAS was applied daily, from the time of admittance to release from the ICU, for a total of 339 measurements. Patients 60 years of age or older were considered elderly patients. The Mann-Whitney test was used to compare the variables.

\section{Results}

The mean age of the patient sample was 70 years. The most frequent type of treatment was clinical $(78.0 \%)$ and most patients were transferred from the emergency room $(40.0 \%)$. The mean stay was 3.5 days and the mortality rate was $38.0 \%$. The mean score for NAS was $66.57 \%(+9.15)$ and remained above the $60.0 \%$ mark for the entire period analyzed. No difference was observed between the mean NAS scores for elderly $(66.44 \%)$ and non-elderly $(66.33 \%)$ patients, just as no difference was observed between the therapeutic interventions carried out in the two groups of patients.

\section{Conclusions}

The results show the need for further discussion on admittance to $\mathrm{ICU}$, regarding the ethical, social and economic implications inherent in intensive care.

\section{PATIENT-CATEGORIZATION IN PRE-HOSPITAL EMERGENCY UNIT \\ M Zunkovic \\ Emergency center, Pre-hospital medical unit, Maribor, Slovenia}

\section{Background}

Categorization of patients by Rush method, intoduced in 1970 in USA, helps us to classify patients according to their needs for special nursing care, to balance effectively the patients' needs for medical resources, provided by the nursing staff in order to control and plan the nursing-staff activities.

\section{Objectives}

Our aim was to classify the needs for nursing care and nursing staff individualy, according to the patients' individual medical condi- 


\section{* Nursing Abstracts from the 2nd Conference of the World Federation of Critical Care Nurses}

tion. The aim was also to introduce this categorization method to our medical unit in order to make the work easier, to improve quality of work and find better solutions to improve quality of healith care.

\section{Methods}

Were included 31 patients from the 15th of April to 22nd of April 2004 and followed the for eight days. All the interventions in each patient were registrated by the Rush method.

\section{Results}

We documented all the interventions performed by the medical staff and afterwards we analyzed them The analysis demonstrated the need for larger number of health care providers to optimize our work and to increase the quality of health care in the field.

\section{Conclusions}

Our investigation demonstrated that the Rush method of categoriztion of patients in the pre-hospital setting is need to organize and to plan the work more efficiently. More research in this field is needed to improve organization and planning in pre-hospital units.

\section{DAILY NEEDS PROFILE FOR NURSING CARE IN AN INTENSIVE CARE UNIT: ANALYSIS BY NURSING ACTIVI- TIES SCORE (NAS)}

KG Padilha ${ }^{1}$, PC Garcia ${ }^{2}$, EMA Finardi ${ }^{3}$, RHK Hatarashi ${ }^{3}$, SCT Bento $^{3}$

1 University of São Paulo - School of Nursing;2 Hospital Universitário - São Paulo;3 Hospital Nove de Julho

\section{Objectives}

The objectives of this study were to characterize the patients hospitalized in an ICU, identify the daily nursing care needs and verify the suitability of human nursing resources to the actual care demand according to NAS.

\section{Methods}

This is a quantitative, descriptive study conducted in a general ICU, with adult patients, in a private hospital in the municipality of São Paulo. The sample was made up of 68 patients, 18 years old or older, who had been hospitalized in the unit for at least 24 hours, and took place in October of 2004. The NAS was collected prospectively, over the period of admission to release from the ICU, from patient records and nurses, for a total of 690 measurements.

\section{Results}

Most of the patients were over 60 years of age, stayed a mean of 12 day in the ICU, were transferred from the surgical unit and were released into semi-intensive care Unit. The NAS mean was $63.6 \%$ $( \pm 2.4)$, and remained above $60.0 \%$ for the entire study. It was observed that, on average, for each 6-hour shift, there was 4.88 hours of nursing shortage. Conclusions

These results contribute to the debate on human resources in ICU nursing and show the need for more research using NAS.

O341 COMPARISON OF PATIENT-CATEGORIZATION BY THE RUSH AND SAN JOAQUIN METHOD IN MEDICAL INTENSIVE CARE UNIT

$\checkmark$ Zunkovic

Intensive Care Unit, General Hospital Maribor, Slovenia

\section{Background}

Categorization of patients by San Joaquin or by Rush method helps us to classify patients according to their needs for special nursing care, to balance effectively the patients' needs for medical resources, provided by the nursing staff in order to control and plan the nursing-staff activities. San Joaquin method, introduced in 1970 in the USA, is more extensively used in Slovenia than the Rush method. Our aim was to compare both categorization methods and to evaluate their efficiency in the real world and every-day clinical work in respect to material resources and staff availability.

\section{Methods}

Included were 29 patients consecutively admitted to medical intensive care unit from 1st of March to the 8th of March 2004 and followed for eight days. All the interventions in each patient were registrated by the Rush and by San Joaquin method and the results of both methods were compared.

\section{Results}

Between the two methods significant differences were observed. The Rush method classified patients more efficiently, regarding the patients' needs for nursing care and therefore the needs for the nursing staff, but also the material resources, needed for quality work in the medical intensive care unit.

\section{Conclusions}

The Rush method seemed to be more objective and precise in categorization of health care than the San Joaquin method in patients in medical intensive care unit.

O347 INSERTION OF NURSES JUST GRADUATED IN THE INTENSIVE CARE UNIT THERAPY: WHAT ARE THEIR DIFFICULTIES AND HOW TO HELP THEM?

S Mori ${ }^{1}$, KJ Nakagawa ${ }^{2}$, IY Whitaker $^{3}$

1 RN, Nursing Department, Universidade Federal de São Paulo Unifesp;2 RN, Intensive Care Unit, Hospital São Paulo;3 RN, MSN, PhD, Nursing Department, Universidade Federal de São Paulo Unifesp

\section{Background}

The Intensive Care Unit (ICU) is characterized for its assistance complexity and the use of specific technological resources. Thus, experienced nurses in the ICU are given priority when employed, but it must be highlighted that the employment of nurses who have recently graduated generates concerns regarding knowledge, assistance and productivity.

\section{Objectives}

To identify the main difficulties reported by the recently Nursing Graduates and Nursing Specialization - Modality - Residence in the ICU, whose first job was in the ICU position and to identify the main subjects analyzed during the course that have contributed to their practices.

\section{Methods}

This is a descriptive study that includes recently graduated nurses from the Nursing Courses between 1999 and 2003 and Specialization Modality - Residence ICU from 1997 to 2003 in Federal University of São Paulo (Unifesp). From the list supplied by Unifesp with 287 names from the Nursing Course and 25 names from Specialization, it was ruled out those already known who did not initially worked in the ICU. To obtain the data, a questionnaire was sent with questions related to their first professional initiation in ICU and the consent form to 175 nursing graduates. 


\section{Results}

Among the Nursing Graduates it was noted that 25 (14.28\%) answered the questionnaire, of these ones 19 (76\%) had begun professionally in ICU in the University hospital. 15 (60\%) answered that the ICU was not the area of their first choice. The main difficulties were regarding to: how to handle the equipment (20), technical assisting skills (16), interpersonal relationship (12), management (11) and the theoretical knowledge (7). The nurses quoted that the subjects which have helped them most in their practice have been: states of shock, advanced cardiac life support (ACLS), neurological evaluation, respiratory failure and mechanical ventilation. As to the Specialization Graduates it was observed that $15(65,21 \%)$ answered the questionnaire. Of these, 10 (58.82\%) had been hired in the ICU of the University hospital. Among the main difficulties quoted we can mention: management (10), how to handle the equipment (5) and interpersonal relationship (4). The subjects quoted as the most helpful in their practice were: shock state, mechanical ventilation, neurological evaluation, respiratory failure, electrolyte regulation, renal failure, dialysis therapy, disorders of glucose, ACLS and poisoning. Actually the theoretical knowledge does not guarantee the practice, but the practice cannot exist without the solid theoretical knowledge. In a wider view this context may have influenced the difficulties among the graduates.

\section{Conclusions}

The results support the importance of directing the nursing teaching in ICU in order to develop better nurses' skills during their education. The nurses have to develop a solid base knowledge in order to be able to process the critic thought, to deal with technology and to accomplish the clinical judgment in ICU environment.

\section{CHECKING AND MEASUREMENT OF NURSING ER- RORS IN ICU}

S Portella, A Carta, LM Contrin, G Simonato, MV Caldeira, L Beccari, MRL Jabur, SMA Lobo

Hospital De Base

\section{Background}

Critical-care nurses search the quality of nursing assistance through the improvement of their work. The processes carried out by the staff must be monitored and measured in order for them to offer opportunities for assistance improvement.

\section{Objectives}

The objective of this prospective study is to measure and quantify the errors made by the General Surgical Clinical ICU nursing staff of a 24-bed hospital school. The data collection was carried out from September 2004 to January 2005.

\section{Results}

A total of 400 patients were enrolled in this study, and 514 error evaluations were performed, totalizing 550 errors related to medications and nursing procedures. Out of these, $41.94 \%$ of occurrences took place in the morning, $40 \%$ at night, and $44 \%$ in the afternoon in the period mentioned. Medication errors were related to unedifying drugs (43.47\%), prescribed and not undertaken medications (23.18\%), schedules errors (17.39\%), and drug administration $(2.17 \%)$. As for the procedures not undertaken in the research period, $33.98 \%$ of the errors were related to undefined serum catheters; $21.35 \%$ to the incorrect fluid balance; $15.29 \%$ to the vesicais probes without attachment, and $10.92 \%$ to past due serum catheters that have not been changed.

\section{Conclusions}

We can conclude that it is important to know and to analyze the errors that might happen, so that interventions and permanent education could be considered in order to sensitize the staff about the problem and to afford medical assistance with quality.

\section{TRANSTHORACIC INTRACARDIAC CATHETERS IN CHILDREN SUBMITTED TO CARDIAC SURGERY: COM- PLICATION DURING THE USE AND THE WITHDRAWAL}

R Mantovani, MN Chaud, MLG Pedreira, WB Carvalho

Federal University of São Paulo

\section{Background}

The hemodynamic monitoring is often an essential issue during the intensive care provided to children submitted to congenital heart disease surgery. Transthoracic intracardiac catheters implanted during the surgery can be useful for vascular pressure gradient measurements in the post operative period.

\section{Objectives}

This study aimed to verify complications related to the use and withdrawal of transthoracic intracardiac catheters implanted in children.

\section{Methods}

Retrospective and descriptive study comprised a population of 88 medical charts of children who had received the implantation of transthoracic intracardiac catheters during the surgical intervention, and that had carried through the postoperative period in a pediatric intensive care unit of a university hospital located in the city of São Paulo, in the period of January, 1998 to July, 2003.

\section{Results}

The results showed that the majority $(61.4 \%)$ of the children was female, with an average of $2.4( \pm 2.5)$ years of age and had been submitted to surgery for the total or palliative correction mainly of Ventricular Septal Defect (20.5\%), Tetralogy of Fallot (18.2\%) and Double Outlet Right Ventricle (13.6\%). A total of 132 transthoracic intracardiac catheters had been identified, being 59.1\% implanted in the Left Atrium, 31.8\% in the Pulmonary Artery, and 9.1\% in the Right Atrium. All the catheters were four French of diameter, manufactured in polyurethane, and $98.5 \%$ had one lumen. Beyond the monitoring purpose, $44(33.3 \%)$ transthoracic intracardiac catheters had been used for infusion of drugs and blood collection. The stability of the patient $(52.3 \%)$, presence of complications $(32.5 \%)$ and death (15.2\%) had been identified as reasons of transthoracic intracardiac catheters use interruption. The study of the complications related to the use of the transthoracic intracardiac catheters identified: blockage (46.5\%), displacement of the catheter $(23.2 \%)$, cardiac tamponade (9.4\%), catheters non-function (9.3\%), emptying $(9.3 \%)$ and cardiac arrhythmia $(2.3 \%)$. The main complications observed during the catheters' withdrawal, were resistance to the withdrawal $(52.2 \%)$ and the bleeding $(30.5 \%)$, most frequents in transthoracic intracardiac catheters of left atrium (65.3\%).

\section{Conclusions}

Complications were present during the use of transthoracic intracardiac catheters mainly related to catheters' blockage and displacement. Resistance to the withdrawal and the bleeding were the main problems verified during the catheters' withdrawal. 
0361 SEDATION OF CHILDREN IN MECHANICAL PULMONARY VENTILATION: CONCORDANCE BETWEEN CLINICAL ASSESSMENT, COMFORT AND RAMSAY SCALES

DM Kusahara, RC Rego, MLG Pedreira, MAS Peterlini, WB Carvalho

Federal University of São Paulo, São Paulo, Brazil

\section{Background}

One of the most important goals of the pediatric intensive care is to achieve the pain and stress relief caused by treatment interventions or treatment. During mechanical ventilation the achievement of an optimal sedation level can improve results and the child's wellbeing, including the prevention of adverse events. Several scores have been developed for the assessment of sedation level. The COMFORT scale was initially designed to evaluate sedation level of children submitted to mechanical pulmonary ventilation, and currently it is one of the most validated sedation scales for pediatric patients. However, the complexity and time spent for COMFORT application can be discouraging, when it is compared with other easier and faster to apply scores as the Ramsay sedation scale.

\section{Objectives}

The aim of this study was to verify the level of concordance between the sedation levels of ventilated children scored by health care professionals, COMFORT and Ramsay sedation scales.

\begin{abstract}
Methods
A descriptive and correlative study was performed at two pediatric intensive care units of teaching hospitals from the city of São Paulo, Brazil. The sample was composed by 77 ventilated children whose clinical conditions did not request deep sedation or sedation withdraws at the moment of the data collection. The sedation scales were applied by trained observers, and the same time the health care professionals responsible by the child care expressed their clinical opinion about the sedation level. The sedation levels were classified as adequate or inadequate (insufficient/excessive). The data was analyzed through percentage of agreement and Kappa (k).
\end{abstract}

\section{Results}

The results indicated that the children had in average 5.08 years of age (median $=5,6$ years), the majority was female $(57.1 \%)$ and received benzodiazepines (96.1\%) as sedative. The percentage of agreement identified between COMFORT and Ramsay scales was of $80.0 \%$, with a Kappa of 0.59 expressing a tendency to concordance. The health care professional's assessment showed a similar percentage of agreement with COMFORT (47.0\%) and with Ramsay scale $(50.0 \%)$. However, the kappa statistics showed a higher concordance level between the health care professionals, clinical assessment and the scores expressed by the Ramsay $(k=0.66)$ than with COMFORT scale $(\mathrm{k}=0.1)$.

\section{Conclusions}

The results of this study indicated that COMFORT and Ramsay scales had a good level of agreement; the health care professional's assessments showed a poor level of concordance with COMFORT and a good concordance with Ramsay scale. Indeed, health care professionals should use scales to assess ventilated children's sedation level.

\section{VARIABILITY IN THE LOCALIZATION OF EXTERNAL REFERENCE POINT FOR CENTRAL VENOUS PRESSURE MEASUREMENT IN CHILDREN}

MLG Pedreira, ASC Belela, MAS Peterlini, GCG Gentil, DM Kusahara, WB Carvalho

Federal University of São Paulo

\section{Background}

The accuracy of central venous pressure (CVP) monitoring can be influenced by the correct localization and constant use of the same external reference point of the right atrium. It is possible to verify in the literature, as well as in practice, a diversity of external reference points and specific criteria for locating it. The mid-axillary line is one of the most commonly used landmark, as an external reference point for CVP measurements in children.

\section{Objectives}

The aim of this study was to verify the variability among health care professionals and a trained evaluator in locating the mid-axillary line as external reference points for CVP monitoring.

\section{Methods}

A prospective study was performed at a pediatric intensive care unit of a teaching hospital from the city of São Paulo, Brazil. During CVP monitoring, five evaluations performed in the same patient by nursing and physicians staff and one realized by an evaluator were compared, resulting in a total of 120 measurements performed by 44 health care professionals and 24 measurements realized by an evaluator trained to locate the mid-axillary line halfway between the anterior and posterior axillary folds, using a metric parameter to identify it. Data obtained was analyzed by " $\mathrm{t}$ " test, Qui-square, ANOVA and Kruskall Wallis tests with a significance level set at $5 \%$.

\section{Results}

A statistical significant difference $(p<0.001)$ was obtained in the average of variation identified for each studied group (professionals and evaluator). Comparing the variability between the external reference points determinations performed by the professionals, $56(46.7 \%)$ were lower than the ones indicated by the evaluator (variation between -0.5 to -9$), 44(36.7 \%)$ were higher (variation between 0.5 to 4$)$, and 20 (16.7\%) were similar (variation 0$)$. The variability $(p=0.778)$ and the concordance level $(p=0.899)$ were not significantly influenced by the professional category; however, a significant negative correlation $(r=-0,26 p=0,005)$ was verified in measurements variability and length of intensive care experience of the professionals.

\section{Conclusions}

The data demonstrated a significant variation between measurements realized by the professionals and the trained evaluator. The variability was not influenced by the professional category (nursing or physicians staff), although professionals with higher length of experience in intensive care demonstrated a tendency of agreement with the evaluator.

\section{SISE, A TOOL TO SUPPORT THE NURSING SYSTEM- ATIZATION}

FM Soares, CE Roma, CR Ferreira, SP Gonçalves

Caism (Centro de Assistência Integral a Saúde da Mulher)/UNI- 
CAMP Campinas/São Paulo -Brasil

\section{Background}

The nursing systematization is increasingly becoming part of the daily nurses routine, either in the direct assistance to the patient or within the academic life, however, it is a process that stops being dominated, needs daily intimacy and availability for study of its scientific bases.

\section{Objectives}

Based in these needs, our objective was to create a software program that facilitated the process of learning and the use of the nursing systematization.

\section{Methods}

The use of a programming language, used internationally and national and international books as data base; we created a software that provides the user with a complete process of systematization of the nursing attendance.

\section{Results}

The software guide can be used in different ways, providing the nurses with different levels of experience, from beginners to experts. The program supplies each serves as apprentice of the nursing systematization individually or generates all process, depending on the needs of the user.

\section{Conclusions}

Software SISE, works as a useful tool, to accomplish the daily nursing systematization, and it has access in different forms, the regular use of the software allows the user to increase its knowledge on the nursing systematization, and assists on the dissemination of this knowledge.

\section{CRITICAL CARE NURSES EXPERIENCE OF CARING FOR DYING PATIENTS: EXISTENTIAL DISTRESS AND ED- UCATION NEEDS}

SF Prestoy

Kutztown University, Kutztown, PA USA

\section{Background}

The critical care hospital environment is emotionally charged. The patient's sudden illness or death dramatically impacts the family, resulting in a range of grief reactions. The family's ability to cope and adapt to sudden loss has been a focus of numerous studies. Nurses work closely with the patient and family and are the primary care givers. The impact of the patient's death and the family's grief on the nurse however, has not been a major research focus.

\section{Objectives}

The major objective of this study was to explore the critical care nurses' lived experience of caring for dying patients.

\section{Methods}

A phenomenological /case study method was used to analyze 40 case studies submitted by 14 registered nurses who worked in critical care units including ICU, NICU, SICU, CCU, and the ER. An open-ended research question asked study participants to describe a professional experience with a dying patient and family, including the nurse's feelings, reaction and adaptation. Case studies were shared in writing with the researcher, and many were also discussed individually or in a group setting. This research sample was a subset of a larger study that examined nurses' lived experience of caring for dying patients.

\section{Results}

Major findings could be divided into two main thematic areas. The first discovered that nurses experienced Existential Distress, questioning why certain particularly tragic or untimely deaths had occurred. Also included in this thematic category, many of the reported death cases emotionally impacted the nurses, to the point where deaths were recalled in vivid detail, and were accompanied by nurses' feelings years later. The second major thematic finding involved the need for education and training, particularly in communicating with the dying, their families, and physicians.

\section{Conclusions}

The findings of this study suggest that hospital and nursing administration may need to address the emotional needs and occupational stress caused by caring for dying patients. Also, hospital educational departments may need to assess educational needs of nurses who provide care to the dying, and provide specific programming related to communication.

\section{THE USE OF IV ADDITIVE DISPENSING PIN FOR ASPIRATION OR INJECTION - AN INFECTION RISK?}

J Henriksen, E Lingaas, M Svendberg

Rikshospitalet Norway

\section{Background}

Some sterile medication comes in multi-dose containers. But is it possible that repeatedly aspirations of these sterile containers may lead to a bigger risk of contamination. To reduce the risk of contamination, we often use "mini-spike plus", an IV additive dispensing pin for aspiration or injection. We wanted to find out if the sterile medicine containers get contaminated with the use of a "mini-spike Plus".

\section{Methods}

"Mini-spike Plus" was used on multi-dose containers with 50 and $100 \mathrm{ml}$ of normal saline $0,9 \%$ (NS). Each container was used by one patient only. It was used for multiple aspirations of NS, drawn out with a sterile syringe as needed, in a period of no longer than 24 hours. Each withdrawal was registered and counted. After 24 hours a sample was taken from each "mini-spike Plus". These samples were cultivated on a medium consisting of blood or chocolate. The containers were added a growth-promoting bouillon, incubated at room temperature for 5 days and after this another 5 days at 37 C. All the procedures where done on a sterile bench, with sterile coat and gloves. The IV additive dispensing pin was kept on the container during the examination.

\section{Results}

110 containers were examined. (42 with $50 \mathrm{ml}$ and 68 with 100 $\mathrm{ml})$. We found growth in 2 of the multi-dose containers $(1,8 \%)$. From the first one of these we also found growth of the same bacteria (staphylococcus aurus) around the IV additive dispensing pin. In the second we found a mix of Staphylococcus hominis and Micrococcus luteus in the container, but no growth in the IV pin.

\section{Conclusions}

What we found indicates that there is a risk for contamination using IV additive dispensing pin. We tried to minimize the chance for contamination at the laboratory by using sterile procedures. 


\section{* Nursing Abstracts from the 2nd Conference of the World Federation of Critical Care Nurses:}

Therefore we assume that our results show real contamination and we have gone through all our routines by using IV additive dispensing pins and changed some of our usage. In our search we added a growth-promoting bouillon into the NS containers, for optimal growth of the microorganism. In normal saline 0,9\% the growth conditions are poor and the chances that the microorganism can multiply are low. Contamination probably happens because: the IV additive dispensing pin construction allows finger touch. The tip of the siring may be contaminated before aspiration from the multidose container. It is probably a bigger risk when the same siring is used more than once. The air that is let into the container can be contaminated. The IV additive dispensing pin can be displaced, in or out, of the container's rubber-cork during usage.

\section{INVESTIGATION OF CHILDREN'S MAIN NURSING PROBLEMS ADMITTED TO THE PEDIATRIC INTENSIVE CARE UNIT: CASE REPORT}

G Tayar, RP Santos, DP Dal'Ge

Instituto Israelita de Ensino e Pesquisa Albert Einstein. Albert Einstein Hospital, São Paulo / São Paulo, Brasil

\section{Background}

In pediatric intensive care units (PICU), the nurse performs the assistance for children with different kinds of pathology, however in order to this assistance be holistic, individualized, and humanized since the admission in the PICU; the nurse needs to perform a precise investigation of current and potential nursing problems developed by the child. They should be in the nursing prescription, verified in the assistance, with the purpose of avoiding and minimizing traumas, and physical and psychic sequelae. The investigation of the child's problems takes part in the systematization of nursing assistance, being data for the selection of nursing interventions aiming at reaching the results which the nurse is responsible for. This procedure allows the plan, co-ordination, and evaluation of actions prioritizing the client assistance.

\section{Objectives}

This paper had the goal to identify the main nursing problems verified by the nurses in the pediatric intensive care unit during the child admission.

\section{Methods \\ The methodology of this paper was performed in two phases. In the first phase, there was a bibliographical study based on Lilacs and Medline. In the second one, the verification of admitted child re- port was conducted in the pediatric intensive care unit of a private hospital in São Paulo city, in the period from July 2004 to January 2005. \\ Results \\ From 46 analyzed reports were verified 22 nursing problems. The most frequent incidents were falling $(100 \%)$, alterations of breath- ing pattern $(73,9 \%)$, pain $(69,5 \%)$, risk of infection related to cen- tral or peripheral venous catheter $(67,3 \%)$, potential pressure ul- cer development $(47,8 \%)$, possible alteration of conscience level $(45,6 \%)$, risk of altered nutrition $(39,1 \%)$, risk of broncho-aspira- tion and haemodynamic alteration $(34,7 \%)$.}

\section{Conclusions}

We conclude that through the investigation of children's main nursing problems admitted to the PICU, it was possible to define the clients' profile, and, consequently, to improve and to specialize the nursing assistance.

\section{DOMESTIC VIOLENCE AGAINST CHILDREN AND TEENAGERS: DOCTORS' AND NURSES' KNOWLEDGE}

CB Fran, MJ Hara

Universidade Federal de São Paulo, Escola Paulista de Medicina - UNIFESP/EPM

\section{Background}

Violence and accidents are a severe and huge problem for public health in Brazil and it is a challenge for preventive education. Violence, in particular, is a universal sociopathy happening with a major or minor incidence in all countries. It overcomes geographical, socio-economic, religious, and ethical boundaries. Crimes are frequent in all societies and they must be seen as a negative aspect of human history. Violence is a complex phenomenon, because its reasons are multifactorial and hard to define. It is based on several areas of knowledge. However, either intrafamiliar or institutional violence play a role of great impact on the child and adolescent victims' health and life quality.

\section{Objectives}

This study had the goal of verifying doctor's and nurse's knowledge about some domestic violence aspects against children and teenagers of a health institute.

\section{Methods}

We performed a descriptive study in a State Research Hospital of secondary level and high complexity, located in a county of São Paulo State. Data was collected through the application of a questionnaire to 55 professionals, doctors and nurses, in four different shifts of the Pediatric units, PICU, and Neonatal ICU. The questionnaire consisted of open semi-structured and structured questions related to professional category.

\section{Results}

$36(65.5 \%)$ doctors and 19 (34.5\%) nurses were interviewed. Their mean of graduation time was 07 years for the doctors and 02 years for the nurses. Most of the right answers were presented in the questions on notification and legal denunciation of suspect or confirmed cases, corresponding to $98.2 \%$ of the right answers in both questions. The question most wrongly answered was related to the kinds of domestic violence known, with $87.3 \%$ of errors. It was observed, also, that there was no meaningful statistically difference between the right number of analyzed questions and the level of professional education with or without post graduation.

\section{Conclusions}

Nowadays domestic violence can be considered as an important compound of the cultural violence of a society, because it already takes part in our social outlook. It was observed that the interviewed professionals are conscious about the notification and legal denunciation. However, generally, in specialized literature the professionals who assist children and teenagers agree, with consensus, that a "sub-notification" of domestic violence cases implicate an underestimation of the real number of theses cases. 
Besides, it makes a strategic plan of prevention in public politics and health advancement difficult.

\section{O400 PROPOSAL OF AN ALGORITHM FOR DETERMINA- TION OF DRESSINGS, SECOND TYPE OF OPEN INJURY IN CHILDREN}

G Tayar, MA Peterlini, MLG Pedreira

Universidade Federal de São Paulo - Escola Paulista de Medicina, UNIFESP/EPM, São Paulo/SP, Brazil

\section{Background}

The wound treatment is part of the nurses' duties. The performance has been increasing progressively in the last years, due to the growing knowledge related to the skin cicatrisation process and the technological and scientific development of nursing cares attending children with many kinds of skin injury. During the post-graduation in pediatrics, the directing of nursing care for children with open wounds and the success of suitable treatment that some of the professionals took in this same institution, when certain dressings were used, made us think about standardizing this care, through the elaboration of a protocol to guide and to support the nurses' decision in the determination of suitable dressing and treatment for children with open injury.

\section{Objectives}

This paper aimed at elaborating an algorithm to support the nurs$\mathrm{es}^{\prime}$ decision in the determination of treatment in different kind of children's wounds and to evaluate its effectiveness according to the pediatric nurses' opinion.

\section{Methods \\ We performed a descriptive study in three phases. The first phase was a bibliographical study of physiology and histology of the skin; the cicatrisation process and the different types of dressing. We previously decided that this paper would be based on: Lilacs, Medline, Cochrane, CINAHL, and bibliographical study and the last 10-year dissertations. The second phase was the elaboration of an algorithm. There were some determinations in it related to the evaluation of skin and cicatrisation stage, the cleaning of wound and the type of primary and secondary bandage to be used. In the third phase, a questionnaire was developed. It was composed of average related to nurse identification and applicability checking of algorithm in relation with the graphic presentation, easy read- ing, sequence, relevance of description of the type of injuries and dressings and support for the nurses' decision during the dressing choice. The categories of the questionnaire were divided into ex- cellent, good, so-so, and bad. There was space for suggestions. 24 nurses of the pediatric department filled out this form in two of the institutions investigated.}

\section{Results}

The $24(100 \%)$ nurses who took part in the evaluation of algorithm were graduated in a mean period of two years and since then they have been working in pediatric area; $87,5 \%$ had post-graduation and a $100 \%$ have been assisting one to four children with open wounds on the mouth. As regards the evaluation of algorithm, most of them of verified average, the protocol was considered excellent and $95.84 \%$ of the nurses considered important the protocol for the application in the assistance practice.

\section{Conclusions}

The proposed algorithm was created and underwent a reformula- tion after the evaluation of the nurses, who took part in the study. Most of the participants considered important the application of this protocol to support the nurses' decision in the choice of suitable dressings in children with open wounds.

0405 THE APPLICATION OF BISPECTRAL INDEX (BIS) MONITORING IN THE PEDIATRIC INTENSIVE CARE UNIT: NURSING AND TECHNOLOGY

BC Alve, CB Fran, FC Regi, RP Sant

Instituto Israelita de Ensino e Pesquisa - Israelita Albert Einstein Hospital

\section{Background}

The bispectral index (BIS) monitor is an electroencephalographic recording device that generates a single numeric value with clinical data indicative of hypnosis. It was created with the purpose of helping the anesthesiologist during the surgical procedures. The monitor is used to calculate a numeric scale from $0-100$ equaling an electroencephalogram pattern of an awake and alert patient. The electroencephalogram is registered for the bispectral index sensor over the temporal-frontal area of the forehead. The goal is to provide an objective, quantitative measure of the level of hypnosis for all patients.

\section{Objectives}

To report the potential applications of Bispectral Index (BIS) monitoring as a helpful tool in a continue evaluation of sedation of a child in a Pediatric Intensive Care Unit (PICU) associated with sedation score.

Methods: We performed a bibliographical study based on data: MEDLINE and LILACS and the training of the interdisciplinary nursing team on the use of BIS monitor and its application in the assistance for patients undergo continue sedation, barbiturate coma, and mechanical ventilation. It is important to highlight that despite the available literature be limited in the BIS application, there are researches already that show the good performance of monitor BIS in emergency rooms and PICUs.

\section{Results}

The results of this study demonstrate that the bispectral index may be a valid monitor of depth of conscious and deep sedation in the spontaneously breathing child. The bispectral index correlating mainly with sedation scores are objective and easy to use.

\section{Conclusions}

The bispectral index offers an objective, safe and reliable measure of sedation because reducing procedure time. Bispectral index monitoring raises the standard of patient care, and in our view, should be used to augment standard assessment. The real question is whether bispectral index can be used to judge the level of sedation in pediatric patients having noninvasive studies.

0413 FUNCTIONING GUIDELINES OF THE INTERMEDIATE CARE UNIT OF THE HOSPITAL DE CLINICAS CARACAS

Y Morales, Y Torres, E Piña, G D Empaire

Unidad de Cuidados Intensivos, Hospital de Clinicas Caracas. Caracas. Venezuela

\section{Background}

As medical and nursing care progress, the necessity of diversifying the health care services arises. It is thus that Intermediate Care Units 


\section{* Nursing Abstracts from the 2nd Conference of the World Federation of Critical Care Nurses:}

are created. They are controversial in many aspects, such as costs, rentability, functional dependence, structure, equipment, scope of pathologies, etc. These units should be analyzed as an innovative and integral product in line with the hospital of the future.

\section{Objectives}

To evaluate the requirements of assistance, in relation to the availability of human resources, equipment and infrastructure in the Intensive Care Unit (ICU) and Intermediate Care Unit (IMCU) at the Hospital de Clínicas Caracas. The aims of this study are to establish if the patients received assistance according to their needs and to establish guidelines for the adequate functioning of the IMCU.

\section{Methods}

The type of study used was "Feasible Project". For the diagnostics phase, the instrument called TISS was applied to 74 consecutive patients who entered the ICU or IMCU in a period of 30 days (September 2003). All data obtained was recorded on specially designed data sheets. Availability of equipment was also recorded. A copy of the floor plan of both units was also used. A two part questionnaire was designed to determine the feasibility of the project.

\section{Results}

The main diagnosis of admission was post-op, cardiac surgery $(40.5 \%)$, cardiac non surgical followed with $20.2 \%$. The third most frequent diagnosis was neurological conditions (13.5\%). Of the 74 patients, 19 had a TISS of $15-19$, and were assigned to IMCU, 49 had a TISS equal or greater than 20 and were assigned to ICU. 6 had a TISS of less than 6 and were admitted to minimal care. According to the TISS, during admission $69 \%$ of the patients were adequately located, during hospital stay $77 \%$ were correctly located and upon discharge $28 \%$ of the patients were correctly located. The average Length of Stay (LOS) was 2.8 days. When the bed efficiency ratio was related to the 43 patients with TISS corresponding to IMCU at discharge, a need for 4 IMCU beds was estimated. The current infrastructure and equipment fulfill the requirements, with the exception of the absence of a physical separation between IMCU and ICU areas, and the presence of ventilators and monitors with a greater capacity than required for IMCU. The functional guidelines for the IMCU were formulated, they were constituted of 16 paragraphs in 5 chapters.

\section{Conclusions}

There were a significant percentage of patients that was not adequately allocated according to their TISS upon admission, and this number increased at discharge. The lack of patient relocation during stay in the IMCU was also noticeable. Physical separation between ICU and IMCU should only exist to permit the communication for patient transfer. The consulted experts gave a high degree of approval to the functional guidelines of the IMCU.

\section{DEVELOPMENT AND EVALUATION OF A WEB-EN- ABLED COMMUNICATION PLATFORM FOR A NATIONAL INTENSIVE CARE NURSE SKILL-MATCHING TO PATIENT ACUITY STUDY}

A Rischbieth

The University of Adelaide Clinical Nursing, Adelaide, South Australia, Australia

\section{Background}

No study has identified a national description of intensive care nurse skill-matching practices in Australian intensive care units
(ICUs). Although there is substantive literature regarding nurse skill mix and resource utilisation, there is a paucity of evidence related to matching nurse skill to patient acuity in ICU.

\section{Objectives}

To design a web-enabled communication platform and innovative survey tools prior to commencement of a national primary multicentre study on intensive care skill-matching practices. Because 59 ICU nurse managers (NUMS) from all Level 3 Australian ICUs, and more than 700 shift leader ICU nurse clinicians (SLNs), were anticipated to participate in this study, development of a web-enabled system was considered to optimise study rigour, recruitment and data management.

\section{Methods}

Design features of the web-enabled system included: simplicity of use, participant anonymity and security, overall functionality, timeliness of data entry and analysis, clarity of questions, ease of pointof-access, and ability for electronic or hard copy data submission. The Intensive Care Skill Matching Study (ICSMS) website was built with a combination of technologies to optimise delivery of content, interactivity and data integrity. It is served off a Linux server running Apache and Tomcat. The data was stored and accessed using SQL and Microsoft SQL Server 2000. The site was driven by HTML and Java, using both Java Server Pages (JSP) and Class/Servlet technology. An autoform was created to allow for hard copy data entry. So as not to affect the primary study recruitment, pilot participants from one Australian state were selected if they had worked in a Level 3 ICU within the past twelve months but were no longer working in one. There were 11 NUM and 10 SLN participants in the pilot study of the developed system. Empirical and descriptive data were collected.

\section{Results}

The database within the platform provided unformatted data with associated look-up tables to allow data analysis using statistical software. The pilot results identified intensive care nurse skill assessment criteria and systems, patient acuity assessment, and skillmatching practices in ICU, including systems to quantify agency nurse skill level. Results demonstrated positive outcomes in participant anonymity and security, functionality, timeliness of data entry and analysis, clarity of questions, ease of point-of-access, and ability for both electronic and hard copy data submission. Following pilot evaluation minor modifications were subsequently made to the web-enabled platform and survey tools.

\section{Conclusions}

A recommendation to adopt the modified web-enabled communication platform and survey tools for the ICSMS study was made, and this study commenced shortly afterwards in March 2005. Critical pilot evaluation of study methods and innovative tools to be used in multi-centre research is highly recommended in the interests of research study rigour, design, participant recruitment, and data management.

\section{O462 CHEST/RESPIRATORY SYMPTOMS OF CARDIAC TAMPONADE PATIENTS IN JAPAN}

Y Ikematsu

Nagoya U

\section{Background}

Dyspnea or respiratory distress is the most prevalent symptom of 
cardiac tamponade patients. However, respiratory/chest symptoms including dyspnoea and chest pain manifest at a variety of medical conditions.

\section{Objectives}

This study aimed to determine Japanese patients' description of chest/respiratory symptoms related to cardiac tamponade.

\section{Methods}

Retrospective case series chart review

\section{Results}

Sixty-six episodes of cardiac tamponade were found in 56 patients admitted to a university hospital in Japan from 1990 to 2000. Among the 66 cases, forty cases were recorded to have some chest/ respiratory symptoms. "Distressed breathing (kokyu ku: kokyu = respiration, $\mathrm{ku}=$ distress)" was most frequently found (20). Terms possibly connoted to dyspnea were "oppressive chest sensation (kyoubu appaku kan: kyoubu = chest area, appaku = oppression, kan = sensation)" (4), "orthopnea (kiza kokyu: kiza = right up position, kokyu = respiration)" (7), "distressed feeling in chest (mune ga kurushii: mune = chest, kurushii (distressed)" (5), and "chest discomfort (kyoubu hukai: kyoubu = chest area, hukai : uncomfortable) (2)". Other chest or respiratory symptoms were "chest pain (kyoh tsu: kyoh = chest, tsu =pain)" (11), "cough (seki or gaiso )" (8) and "palpitation (dohki )" (4).

\section{Conclusions}

Japanese cardiac tamponade patients complain about their chest/ respiratory symptoms in a variety of terms. Some symptoms are difficult to determine whether they have respiratory or cardiac origin. Comparing the findings to symptoms related to other cardiac or respiratory conditions may contribute to clarify definitive symptoms of cardiac tamponade.

0466 THE CHALLENGE OF INTRODUCING CONTINUOUS ELECTROENCEPHALOGRAPHIC MONITORING TO A GENERAL INTENSIVE CARE UNIT

\section{JL Cook}

Princess Alexandra Hospital, Brisbane, Queensland, Australia

\section{Background}

At Princess Alexandra Hospital (PAH) a review of neurocritical care monitoring approaches was prompted by an expansion in interventional services and increasing neurological admissions. To enhance the management of these patients, senior medical and nursing staff introduced continuous electroencephalographic (CEEG) monitoring. Despite bedside availability, few units in Australia have embraced CEEG technology. The CEEG patient monitoring unit has an electroencephalographic (EEG) module displaying one or more EEG channels and a numerical measure of EEG power spectrum. The Philips CMS system used at PAH intensive care unit (ICU) displays two EEG waveforms and a continuous spectral array. Barriers to implementation included the cost of purchasing and maintaining the equipment, medical and nursing unfamiliarity with EEG waveforms and interpretation, and clinical scepticism regarding the usefulness of the data obtained. This paper examines strategies to minimise the difficulties associated with the introduction of CEEG monitoring. These strategies should be considered when introducing similar new technologies so that they can be successfully implemented.

\section{Objectives}

The objective of this study is to examine the process of implementation of CEEG as a new technology in a general ICU.

\section{Methods}

A case study is presented illustrating the application of bedside EEG monitoring to improve the medical and nursing management of a patient with prolonged status epilepticus. Experiential and reflective practice identified strategies to improve clinical integration of the new technology.

\section{Results}

As anticipated, the key factors were that effective implementation of CEEG monitoring required a coordinated educational effort, commitment to success and a team approach. When faced with technical difficulties and competing tasks of higher priority in the busy environment of a general ICU, staff became easily disillusioned and disengaged with the new technology, preferring to use established management practices.

\section{Conclusions}

This case demonstrates that to achieve effective implementation of new measurement modalities such as CEEG monitoring, barriers to success must be anticipated and addressed, including ongoing education and equipment management issues. Additionally, strategies must be employed to facilitate the inclusion of new technology into existing patterns of clinical care.

\section{A NURSE INITIATED TELEPHONE FOLLOW UP SERVICE FOR FORMER GENERAL INTENSIVE CARE PA- TIENTS}

S Mckinley2, D Moran1, R Elliott2

1 The Royal North Shore Hospital of Sydney;2 University of Technology Sydney

\section{Background}

Intensive care nursing involves the treatment and care of patients with life threatening conditions. Studies investigating patients' recollections provide valuable insight about the intensive care unit (ICU) experience. Patients report various sources of discomfort including unfamiliar obtrusive noise, disturbed sleep, an inability to communicate effectively and the presence of intrusive monitoring devices and tubes. The experience may result in long-term physical and psychological effects, ranging from fatigue and sleep disturbances to post-traumatic distress symptoms.

\section{Objectives}

We describe the process and outcomes of one nurse initiated strategy to address the needs of adult general intensive care patients following discharge from hospital.

\section{Methods}

The intensive care follow up telephone service has been in progress since July 2001. The aims of the service are to offer former patients an opportunity to explore the intensive care experience and provide support during the recovery phase. Patients are phoned 8-10 weeks after discharge and respond to a semi-structured interview, including questions about mobility, sleep, ability to concentrate and relationships and support. Some patients are referred to other services, such a social work and some return for an escorted visit to the ICU.

\section{Results}

Patient experiences vary during recovery but many report physi- 


\section{* Nursing Abstracts from the 2nd Conference of the World Federation of Critical Care Nurses:}

cal fatigue, sleep disturbances and financial difficulties. Patients who have been telephoned say they benefit the opportunity to talk about their experience and receive general health related information about diet and exercise. Patients who visit the unit find it helpful in piecing together their ICU stay. The nurses who have participated in the service have enjoyed greater work satisfaction related to exposure to the patients who are recovering. It offers an opportunity to complete the episode of care. In addition we have been able to feed patients suggestions into existing quality frameworks. The information received from former patients has assisted in the development of a home based physical rehabilitation program, which will be evaluated using a randomised control trial. Conclusions

The follow up service positively contributes to patient outcomes and the profile of critical care nursing in the community.

\section{ASSESSMENT AND REDUCTION OF ANXIETY IN MECHANICALLY VENTILATED PATIENTS}

S Mckinley ${ }^{1}$, L Dean $^{2}$

1 University of Technology Sydney;2 Childrens Hospital Westmead

\section{Background}

Critically ill patients report that they experience psychological distress, including anxiety and fear, during treatment in an intensive care unit (ICU). Investigators are increasingly seeking to learn about patients' anxiety levels during critical illness. Clinical practitioners seek to avert and alleviate anxiety and fear in their patients in order to promote comfort during ICU treatment. Anxiety may also be associated with physiological responses that interfere with patient recovery and contribute to increased morbidity and mortality observed in more anxious critically ill patients. Thus the detection and reduction of anxiety in critically ill patients are important clinical goals.

\section{Objectives}

To i) review approaches to the assessment and reduction of anxiety, and ii) evaluate an information intervention to reduce anxiety during repositioning (turning) in mechanically ventilated patients.

\footnotetext{
Methods

i) A comprehensive review of published research on assessment and reduction of anxiety in ICU patients. ii) A pilot randomised controlled trial (RCT) of provision of information containing the sensations experienced during repositioning was conducted in 40 mechanically ventilated patients. Patients were assigned to have information about the turn provided according to a control $(\mathrm{C})$ script that contained minimal information about sensations, or an intervention (I) script that provided detailed sensory information about what would be experienced. Anxiety was measured before and after the turn using the Faces Anxiety Scale.

Results

Anxiety has been assessed by objective observation of physiological and behavioral signs or by patient self report of the extent to which they are feeling anxious. As objective signs of stress are difficult to interpret and potentially unreliable in critically ill patients, research into patient anxiety in ICU relies on patient self report. Investigators have used measures such as the Hospital Anxiety and Depression Scale, the Edmonton Symptom Assessment Scale, and the six item version of the Spielberger state anxiety scale (SAI). However critically ill patients are limited in responding to such anxiety scales, especially ventilated ICU patients. The Faces Anxi-
}

ety Scale is a single item scale with five possible responses, scored from 1 to 5, to which more ICU patients are able to respond than to other brief scales. It has good criterion validity vs. trained clinical judgement of anxiety $(r=.64)$ and vs. the SAI $(r=.64)$. In the pilot RCT the mean (SD) anxiety levels prior to turning were $C-2.5$ (1.1), I- 2.6 (1.1) and after the turn were C $-2.5(1.1), I-2.35$ (1.2) $(\mathrm{F}=0.24, \mathrm{p}=.63)$.

\section{Conclusions}

Anxiety is difficult to assess in mechanically ventilated patients but the Faces Anxiety Scale provides a valid way to do so. Provision of information about sensations experienced during turning in this small pilot study did not significantly reduce state anxiety. More intensive interventions may be required to reduce anxiety in this patient population.

\section{CONCEPTS AND ATTRIBUTES USED BY CRITICAL CARE NURSES TO MAKE SEDATION RELATED DECI- SIONS}

LM Aitken $^{1}$, A Marshall ${ }^{2}$, R Elliott $^{3}$, V Fox $^{3}$, S Mckinley $^{2}$

1 The University of Queensland, Brisbane, Queensland, Australia;2 University of Technology Sydney, Sydney, New South Wales, Australia;3 Royal North Shore Hospital, Sydney, New South Wales, Australia

\section{Background}

Sedation of patients forms an integral aspect of management of the critically ill patient. There is widespread agreement that the adequate level of sedation for each patient is different and is based on factors such as clinical condition, current management goals and history. Despite this recognised variation, there has been little attempt to understand how the critical care nurse determines how much sedation is required and when to deliver it.

\section{Objectives}

This study was designed to describe the concepts and attributes that critical care nurses use when making decisions related to assessment and management of a patient's sedation requirements

\section{Methods}

Seven critical care nurses participated in the study including two pilot participants. The remaining five critical care nurses had data collected on two occasions while they cared for a general intensive care patient. Participants were not aware that sedation practices were the specific area of study focus. Two methods of data collection were used including thinking aloud and observation, with follow-up interviews conducted to elicit further detail and rationale regarding decisions made. Analysis involved integration on the data from all three collection sources (thinking aloud, observation and interview). Protocol analysis was conducted to code the attributes and concepts used to make decisions related to sedation practices. The study was approved by relevant Human Research Ethics Committees.

\section{Results}

Four female and three male critical care nurses, with between 5 and 25 years nursing experience participated in the study. Participants had between 4 and 23 years critical care experience and all had a hospital or university based critical care qualification. Each participant organised their decision making around five to nine concepts relating to sedation assessment and management. Concepts could be categorized as either assessment, treatment or pathophysiologi- 
cal in nature. Concepts were more frequently treatment oriented, with 26 treatment concepts, 20 pathophysiological concepts and 16 assessment concepts identified. The most common concept in each category was: treatment - sedation, pathophysiology - anxiety, assessment - assessment of response or neurological assessment. Anxiety and agitation appeared to be used interchangeably.

\section{Conclusions}

Extensive data are used by critical care nurses when making sedation decisions. These data relate to treatment, pathophysiology and assessment, with an emphasis on treatment. Description of these data will allow improvement in educational and quality assurance programs related to sedation in order to improve this area of practice.

\section{FAMILIES' PERCEPTION ON COMMUNICATION IN AN INTENSIVE CARE UNIT}

K Kitajima, M Cosmo, M Rodrigues, P Godoy

Hospital da Venerável Ordem Terceira da Penitência

\section{Background}

Communication with families in intensive care setting can be complicated by the fact that admission is often sudden and unexpected and intensivists usually meet families in difficult emotional circumstances. (Critical Care Medicine, 2001).

\section{Objectives}

Identify families' perception about information in intensive care unit (ICU)

\section{Methods}

It is a qualitative and prospective research. Thirty families responded to a semi-structured questionnaire involving topics about phone information in the morning and communication with the intensivists. The patients had to be in the ICU for at least twenty four hours. Data was collected by psychologists in a period of a week.

\section{Results}

About patient: average age was 78 years old; time of internment in ICU was five days and $43 \%$ were of son or daughter. About information obtained by phone: $60 \%$ do not use this service, due to its proximity to visit period $(44 \%)$. In relation to the content of the information: $63 \%$ find important to receive general information on the patient, such as evolution and intercurrence. In relation to the communication with the intensivists: $63 \%$ are satisfied with the relationship with the health team, nothing to complain in communication $(40 \%)$. In relation the suggestions to improve the communication: $26 \%$ suggest that the language must be simpler; $20 \%$ must always say the truth and $20 \%$ that the intensivists would have to give more attention.

\section{Conclusions}

With these preliminary results, we may conclude that the families have a great necessity of information, independent of age, diagnostic and time of internment of the patient in the ICU. Communication in the ICU requires well-trained professionals in the use of language and with an empathic attitude to understand better the families' needs, besides an institutional culture that promotes a good relationship among all the staff with families and patients.

\section{INTER-HOSPITAL TRANSPORT IN CRITICALLY ILL PATIENTS. FIVE YEAR EXPERIENCE}

I Fuentes, A Donoso

Area de Cuidados Criticos Hospital Padre Hurtado Santiago Chile

\section{Background}

Pediatric intensive care patients often need to be moved from critical care areas to another units for diagnostic and therapeutic procedures; specially in General Hospital without integral solving capacity.

\section{Methods}

In order to assess the problem encountered during the inter-hospital transport (IHT) procedure, we elaborated a prospective study. We elaborated a categorization according to the patient's complexity in order to determine medical transport team. Each medical team can be formed by critical care physician, critical care nurse and paramedics. A questionnaire was undertaken to evaluate the transport of critically ill children hospitalized in our PICU over a five years period (1999-2004).

\section{Results}

Two hundred eleven children transfers were performed. All transfers were made in normal emergency ambulances. The most common cause of transfer was medical evaluation. 91\% IHT happened during the day and the average time spent was 122 min (range: 30-450 min). The transfer team was integrated exclusively of paramedicals in $37 \%$ and only $26 \%$ of physicians. $31 \%$ in mechanical ventilation. $14 \%$ receiving at least one medication (mainly sedatives). All ventilated patients were supported manually. Deterioration in respiratory systems was the main complication in the patient's condition and the main inconvenient indicated by the transfer team was administrative problems. No cardio respiratory arrest and death were observed. No differences in adverse event occurrence according staff composition were observed.

\section{Conclusions}

IHT is usual in Pediatric Intensive Care Unit and can be achieved safely and effectively with adequate facilities and staff. Categorization was useful in order to optimize resources. Transfer procedure will always pose a risk, but this can be minimized by adopting recommendations.

\section{NURSE THEORETICAL TRAINING FOR THE ASSIS- TANCE TO A CARDIOPULMONARY RESUSCITATION}

M Bellan Consorti, IE Araujo Muglia

Universidade Estadual de Campinas - Unicamp

\section{Background}

A cardiac arrest is a situation that occurs most of the time in a sudden and unexpected way. Due to the fact that the nursing team is closer to the patients, it is them who detect such events and should have enough information to provide first assistance. The success of this cardiopulmonary resuscitation (CPR) depends on the starting time, the harmony, the team's synchronism and the ability of the professionals involved.

\section{Objectives}

The objectives of the study were to offer a theoretical training to nurses to assist cardiac arrests (CA) and check the information before and after the participation in the proposed activity. 


\section{* Nursing Abstracts from the 2nd Conference of the World Federation of Critical Care Nurses}

\begin{abstract}
Methods
A theoretical kind of program was applied according to the directories published by GUIDELINES 2000 FOR CARDIOPULMONARY RESUSCITATION AND EMERGENCY CARDIVASCULAR CARE - IN INTERNATIONAL CONSENSUS ON SCIENCE to the nurses who develop assistance activities in the various areas of the hospital. This program covered two phases, the first one related to the application of a questionnaire on CPR followed by a theoretical class on the subject. The second phase, done a week after the first one, covered a reapplication of the questionnaire on CPR to check the level of assimilation of the subjects discussed during the theoretical class.
\end{abstract}

\section{Results}

Forty nurses participated. On the first phase, six (15\%) of the participants got grades between 0 to 4,9 and after a week, two (5\%) of them got grades between 5,0 to 7,0 and four (10\%) between 7,1 to 10,0 . Of the ones who got grades between 5,0 to 7,0 , nineteen $(47,5 \%)$ of the participants in the first phase, a week after, eighteen $(45 \%)$ of them presented a grade between 7,0 to 10,0 . And fifteen $(37,5 \%)$ of the participants got a grade similar to or over 7,1 on the first phase and after a week they maintained this grade.

\section{Conclusions}

Offering a program with theoretical subjects on CPR resulted in nurse's improvement of knowledge on the assistance to CPR and would also subsidize them in organizing and orienting their team as well as their own professional performance.

\section{AUDITING UNPLANNED EXTUBATION IN AN AUS- TRALIAN CRITICAL CARE UNIT}

KM Birkett1, KA Southerland1, GD Leslie2

1 ROYAL PERTH HOSPITAL, PERTH, WA, AUSTRALIA; 2 Edith

Cowan University, Perth, Wa, Australia

\section{Background}

The majority of patients in Australian Intensive Care Units (ICU) are intubated and ventilated, thus exposing them to numerous potential life threatening complications such as laryngospasm, laryngeal oedema, aspiration pneumonia, arrhythmias, bronchospasm or respiratory failure. To monitor the incidence and outcome of Unplanned Extubation (UE), a clinical indicator was established in 1995. Various quality improvement projects have since been evaluated.

\section{Objectives}

The aim of this particular audit was to determine the frequency and risk factors associated with reported UE within a 22 bed general and surgical ICU between January and December 2003.

\section{Methods}

Nursing and medical staff provided information on the patient's age, diagnosis, mental status, precipitating causes and investigations/treatment ordered. Other data collected included information on the patient's position, sedation regime, method of ETT placement and the use of physical restraints.

\section{Results}

Results indicated that twenty six patients experienced an UE in 2003 (incidence of 2.04 per 100 patients), a finding consistent with previous years $(2.74 \%)$. Of these, $46 \%$ of these required emergency reintubation most of which were accidental extubations (71\%).
Unplanned extubation was generally deliberate (73\%), occurred in general patients (85\%) and between the hours of 2100 and 0600 $(42 \%)$. Other findings revealed that UE took place despite the provision of sedation or restraints.

\section{Conclusions}

Practice review should focus on reviewing tube fixation methods, developing weaning protocols for general patients and assessing an appropriate sedation score to monitor the patient's mental status. Following implementation of each review, further audit should be undertaken to evaluate outcome. Overall, data collected from this and previous surveys revealed an encouragingly low incidence of UE. The UE indicator provides the opportunity to monitor changes and guide further clinical practice.

\section{NURSING ACTIVITIES SCORE (NAS): AN APPLICA-} TION METHOD

LA Gonçalves1, KG Padilha2

1 Hospital Universitário da USP;2 Escola de Enfermagem da USP

\section{Background}

NAS is an instrument used to measure the workload of nursing staff in the Intensive Care Unit. The difficult faced in filling out the instrument and the necessity of making its application available led us to develop this proposal.

\section{Objectives}

To standardize NAS application, through language standardization among ICU nurses and adapt the time consume spent to carry out particular items according to the reality.

\section{Methods}

The study was carried out in an ICU in a Med-school in the city of São Paulo (Brazil). 13(72.2\%) nurses took part of the standardization phase in NAS daily data collection. At first, meetings were carried out in order to a better familiarization with the instrument. After that, each nurse recorded in a form, the estimate time spent to realize NAS sub-items concerning time in either 6 or 12-hour duty. Based on that, the average time spent with relevant items was calculated.

\section{Results and Conclusions}

The methodology applied enabled us to design NAS-AMENDMENT form with time standardization to carry out nursing tasks by work shift (morning, afternoon and night) particular to that ICU. Besides that, each item in the instrument that contributed to a more reliable data collection was operationally defined.

\section{RISK FACTORS FOR INTENSIVE CARE UNIT (ICU)} READMISSIONS

BFC Santos, OFP Santos, LR Guastelli, CR Laselva, M Oliveira, M Cendoroglo, E Knobel

Hospital Israelita Albert Einstein

\section{Background}

The unexpected hospital readmissions, especially in ICU, determine increase of the hospital costs and larger mortality for the patients. However, a significant number of readmissions are potentially avoidable. Thus, the interest in the study of the factors involved in the non scheduled readmission is driven by the hypothesis that the improvement of the care can result in a reduction of the readmissions, with consequent benefits for the patients and 
reduction of the cost of the treatments of health.

\section{Objectives}

To identify risk factors for the patients' readmission in ICU.

\section{Methods}

We accomplished a study cohort, based on data of patients' interned in ICU. The internments of January 01, 1999 were analyzed to December 31, 2000 in UTI. We established two groups, the first group was composed of the patients that just presented an internment, and the second group was composed of the patients that presented two internments in UTI during the same hospital internment. For the analysis the first internments of the two groups were analyzed.

\section{Results}

In the study period 3,034 were interned patient in UTI. In this population, the readmission rate in UTI was of $10.7 \%$ and the average occupation was of $86.76 \%( \pm 4.16)$. The readmitted patients presented age higher average $(67.5 \pm 15.5$ years vs $63.2 \pm 17.2$ years ; $<$ <.0001). The APACHE II and SAPS II higher in the readmitted group 18.7 (6.4) and 33.1 (12.9), in the group it controls 12.0 (6.3) and 27.7 (11.9) respectively, both with significant difference. The hospital and ICU LOS presented significant difference among the two groups. In the multivariate analysis, the presence of, APACHE II $>17$ ( $p<0.0001$; OR 4.14; IC 95\% 3.16 - 5.44), creatinine $>2.0$ in the moment of the internment ( $p<0.0507$; OR 1.56; IC 95\% $0.99-2.42)$, and ICU LOS $>5$ days ( $p<0.0426$; OR 1.42; IC $95 \%$ 1.01 - 1.98) they presented significant difference and they were identified with independent variables for readmission in UTI.

\section{Conclusions}

In the moment of the discharge of patient UTI that presented time of larger permanence than five days and in its APACHE admission larger II than 17, larger age than 65 years, creatinine larger sérica than two and origin of the step down unit present significant risk of readmission in the $\mathrm{ICU}$.

\section{CASE REPORT: APPLICATION AND MAINTENANCE OF PERIPHERALLY INSERTED CENTRAL CATHETERS IN A PEDIATRIC INTENSIVE CARE UNIT}

RP Santos, AAR Souza, LB Cunha, AS Pereira

Instituto Israelita de Ensino e Pesquisa Albert Einstein. The Department of Maternity and Children. Brasil

\section{Background}

In Pediatric Intensive Care Unit (PICU), frequently, severe sick patients need a complex intravenous therapy, in the most cases, in central circulation. Among the newest techniques of central catheter application, it is the Peripherally Inserted Central Catheters (PICC). The PICC is a central catheter, its insertion is conducted by a qualified professional who have trained to execute this procedure through a peripheral puncture. The risks related to the catheter procedure and maintenance are smaller than in conventional techniques. In this way, we observed a progressive increasing of recommendation and use of this new technique in PICU.

\section{Objectives}

To know the index of success in the application and maintenance of PICC in a PICU.

\section{Methods}

We performed a retrospective study through reports on PICC protocols which were filed. The sample was composed of 41 patients, at the age between 1 and 166 months, who have undergone the application of PICC from March 2004 to February 2005.

\section{Results}

From $100,0 \%$ of catheters inserted by nurses, the indications included antibiotic therapy $(93,6 \%)$, post-operator of big surgeries $(4,9 \%)$, and assistance for chronic patients $(2,5 \%)$. In the application, we had $70,7 \%$ of successes. The mean time of internation was 12 days, the minimum time was one day and the maximum time was 24 days. About the removing of PICCs, 46,3\% happened in the end of therapy and 26,8\% for complications: 4,9\% for obstruction, $12,2 \%$ for rupture, $7,3 \%$ for phlebitis, and $2,5 \%$ for exposing.

\section{Conclusions}

This paper highlights the importance of procedure as alternative to implement intravenous therapy and shows some aspects of improvement in the maintenance of PICCs, that is, actions for the improvement of successful index in maintenance and reinforcement in the prevention of adverse occurrence, resulting in decreasing of premature removing of catheter.

\section{MANAGING THE CHALLENGE TO DELIVER EFFEC- TIVE AND APPROPRIATE INTENSIVE CARE - THE COM- MISSIONING OF A NEW INTENSIVE CARE SERVICE AND THE DEVELOPMENT OF AN INTEGRATED “HOTFLOOR" MODEL}

BJ Abbenbroek

Royal Prince Alfred Hospital, Sydney, Australia

\section{Background}

There is an increasing demand for complex intensive care which is resource intensive and faces the challenges of resource limitations, skilled workforce shortages and escalating costs.

The concentration of intensive care services into large integrated units is emerging as a strategy to meet this challenge by consolidating resources, allowing greater flexibility in the model of intensive care delivered and providing hospitals with the opportunity to become "magnate" units to attract skilled staff. In July 2002 the new Intensive Care Service was commissioned which is a 54 bed integrated critical care unit which incorporated Cardiothoracic ICU, Neuroscience's ICU, General ICU and a new High Dependency Unit. The new "hotfloor" model provided both challenges and opportunities to implement new models of care, operational processes and critical care management. Ongoing monitoring of clinical indicators to evaluate patient, staff and organisational outcomes is critical to the success of this process.

\begin{abstract}
Methods
A comprehensive set of 19 clinical indicators were collected on a daily basis prior, during and following the commissioning of the new integrated Intensive Care Service between January 2001 and June 2005. The indicators provide measures of clinical activity and quality of care characteristics of the service including length of stay, mortality, access block, unplanned readmission's, unplanned extubations and cross infection rates. The clinical indicator data set was evaluated in 6 month intervals over the period studied which coincided with the move to the new intensive care unit in July 2002 providing an opportunity to compare activity and outcomes.
\end{abstract}




\section{* Nursing Abstracts from the 2nd Conference of the World Federation of Critical Care Nurses}

\section{Results}

A significant increase in activity occurred following the move to the new unit however reductions in the average length of stay, mortality, admissions refusals, access block, unplanned readmission's, mechanical restraints, pressure areas and cross infections were demonstrated.

\section{Conclusions}

The building and commissioning of a new integrated Intensive Care Service, which utilised the "hotfloor" concept, provided the opportunity to implement innovative models of intensive care and new management models. A positive impact on both organisational and patient outcomes was demonstrated. The clinical indicator data set provided a valuable and unique opportunity to evaluate the impact of these strategies and the effectiveness of the change management processes used to facilitate the commissioning of the new unit. Large integrated intensive care services provide an increased opportunity to manage the demands and resource limitations that challenge the effective and appropriate delivery of intensive care.

\section{ACCIDENTAL EXTUBATION IN A PEDIATRIC INTEN- SIVE CARE UNIT}

RP Santos, SB Marchini, V Sekkel

Instituto Israelita de Ensino e Pesquisa Albert Einstein. Department of Maternity and Children. Albert Einstein Hospital, Sao Paulo, Brazil

\section{Background}

The endotracheal intubations and the mechanic ventilations are routine procedures in pediatric intensive care units. Despite the technical procedures to be established, there are daily inherent risks in children and adolescent care that can still lead to the accidental extubation. The accidental extubation can be defined as a not planned extubation. If ventilation is inadequate or absent, the nurse begins to ventilate the child using a bag-valve mask with a reservoir and high-flow oxygen. This event can take to hypoxemia, bradycardia and in some cases it can cause death. All not planned extubation, that could have caused iatrogenic damage for the patient, must be analyzed to unravel the causes, in order to prevent the persistence of an error in the nursing care process. Albert Einstein Hospital is a general hospital in São Paulo - Brazil, with 420 beds. The PICU has 9 beds, for patients from neonates to adolescents. The accidental extubation was treated like an adverse occurrence and could be detected by any professional of PICU.

\section{Objectives}

The aim of this study is to present the construction of a quality indicator for the process of maintenance of the endotracheal intubations in critically ill children.

\section{Methods}

Descriptive study. Monitoring of the number of iatrogenic extubation from August 2004 to January 2005. Goal: absence of iatrogenic extubation and original causes.

\section{Results}

In the studied period we found: August: $0: 19$ patients intubated/day; September: 0 : 18 patients intubated/day; October: 1 (not planned extubation) : 26 patients intubated/day; November: $0: 28$ patients intubated/day; December: $0: 19$ patients intubated/day; January 2005: $0: 7$ patients intubated/day. Factors that contribute to the occurrence are: age-group (child up to one year), ac- cumulation of secretions, sedation level, inefficient restriction of the members, execution of procedures and inadequate intubation path. However, despite the analysis of the unique occurrence, we can identify clearly the lack of the systematic approach to sedation and the dependence on subjective assessment as barriers to effective sedation.

\section{Conclusions}

Sedation is a necessary part of intensive care and maintaining an optimal level of sedation is an important procedure. Sedation aims to diminish pain and to promote comfort and compliance with routine care and ventilation. Sedation is associated with sudden changes in consciousness, distress, agitation and accidental displacement of lines, intolerance of mechanical ventilation and haemodynamic disturbances. Inadequate sedation contributes to family distress. As we can see the results of the study strengthen the necessity of the intensive care unit to adopt a protocol of sedation and analgesia to suggest the lack of sedation.

\section{PREMATURE NEWBORN PARENTS CONCERNS ABOUT THE DISCHARGE FROM NEONATAL INTENSIVE CARE UNIT}

FS Balbino, VL Barbosa, M Naganuma

UNIFESP - Sao Paulo - Brazil

\section{Background}

Discharge is a moment of too much expectation for parents after long time of treatment in a neonatal intensive care unit. They are now responsible for a small, fragile and almost unknown baby expressing different category of concerns about that.

\section{Objectives}

The aims of the study were to identify and describe the main concerns expressed by parents when the discharge from neonatal intensive care unit comes closer

\section{Methods}

A qualitative, descriptive -exploratory research was implemented in a neonatal intensive care unit at the city of Sao Paulo - Brazil. The data was collected by a semi-structured interview of the parents and by neonates' medical records

\section{Results}

The data analysis allowed the rising of these categories: Concerns about discharge proximity, because, as this moment approaches, parent shows concern about the risk of infection at the hospital or at home; development and growing compromises; the premature baby's future and the new responsibility and coping skills they will have to achieve; and Parents' concern about taking care of the premature neonate, in which they described the great responsibility of child care at home, about breastfeeding and respiratory complications that could compromise the child health or leading to death.

\section{Conclusions}

Facing these results, strategies were proposed to promote parents' participation in their child's care during hospitalization, enabling them to discharge from hospital, minimizing the concerns presented in this moment

0595 LIVING THE MOMENT OF HOSPITAL DISCHARGE OF THE PREMATURE NEWBORN FROM NEONATAL INTENSIVE CARE UNIT: FEELINGS EXPRESSED BY PAR- 


\section{ENTS}

MJ Avena, FS Balbino, VL Barbosa, M Naganuma

Federal University of Sao Paulo - UNIFESP - Sao Paulo - Brasil

\section{Background}

In a neonatal intensive care, the discharge moment is full of emotion and when the parents receive the information they express in a verbal form and in most situations in a non verbal form, a brief moment of happiness and excitement as they are achieving something that could not be possible.

\section{Objectives}

To identify the feelings expressed by parents at the discharge moment of premature newborn.

\section{Methods \\ A qualitative, descriptive -exploratory research was implemented in a neonatal intensive care unit at the city of Sao Paulo - Brazil. The data was collected by a semi-structured interview of the par- ents and by neonates' medical records \\ Results \\ The category that emerged from the parents reports was classified as an emotional dimension, expressed by parents when the mo- ment of discharge happens, distinguishing positive and negative feeling themselves. Thus the parents manifest fear, insecurity, anxi- ety, joy, uncertainty and doubt about the moment that they will be responsible for a baby with such peculiar characteristics, as the premature neonate. \\ Conclusions \\ The premature neonate's discharge may be as insecure as the deliv- ery premature moment and the parents must be prepared as soon the surviving is clinically probable. Some new orientations were proposed in the neonatal intensive care unit to prevent parents emotional distress, promoting parents' participation during the child care, enabling them to face the discharge from hospital, as a normal process, minimizing concerns and promoting confident relationship with the nursing staff.}

0605 DEATH, AND ITS GIFT TO LIFE. A PHENOMENOLOGICAL STUDY OF THE EXPERIENCES OF ORGAN DONORS' RELATIVES DURING THE ORGAN DONATION PROCESS

A Orøy

Molde Univeristy College, Molde, Norway

\section{Background}

This study deals with brain dead and organ-donation, and the relatives' experiences with the organ-donation process.

The purpose of this study was to investigate what the relatives of the pronounced brain-dead patients' experiences during the anticipation, confrontation and post confrontation stages of the organdonation process.

\section{Methods}

The method used is qualitative and phenomenological. The data were obtained by in-depth interviews with eight relatives, from seven different situations. All of them had lost a loved one suddenly. Four of them consented to donation and three of them did not consent to donation. The relatives were interviewed in a period of about 8 month and 2 years after the death occurred, and the interview took place in the family's home. Data were analyzed by
Giorgi's phenomenologically inspired four-step method. The purpose of the study was to discover the most important characteristics of the relatives' experiences during the organ-donation process.

\section{Results}

Findings showed the following process: The beginning of the end; The end; The question; Farewell; The time afterwards; Life goes on; Death, -and it's gift to life.

\section{Conclusions}

Findings showed that the question about using organs' from the brain-dead patient presented an additional problem for the relatives in an already vulnerable situation. The question of organ-donation can not be seen as an isolated phenomenon, but as an integrated part of the organ-donation process. My study further showed that the ability of the personnel to create calmness in a chaotic situation and the attitude of the personnel was of the greatest importance to the relatives. The theoretical framework for the study is phenomenological philosophy and etic in relations.

\section{Reference}

Georgi, A. (1994): Phenomenology and Psychological Research. Pittsburg, PA.Ducuesn Univerity Press.

\section{CARE TECHNIQUES DEVELOPED BY HEALTH PRO- FESSIONALS AT AN INTENSIVE THERAPY UNIT}

LM Andrade1, JA Caetano2, EM Rocha3, RM Pontes4

1 IJF/ UNIFOR;2 UNIFOR;3 IJF;4 SANTA CASA SOBRAL

\section{Background}

An Intensive Therapy Unit is considered nowadays a place where qualified and specialized assistance is delivered. This sector is composed of a range of functionally grouped elements destined to the assistance of risk patients who demand continuous nursing and medical care, apart from specialized equipment and human resources.

\section{Objectives}

This paper aims at understanding the humanized assistance to patients interned in an ITU from health professionals' point of view.

\section{Methods}

This is qualitative character research carried out in the ITU sector of the Irmandade da Santa Casa de Misericórdia de Sobral hospital. The subjects of the study were health professionals who worked full-time at the sector in June 2004, of which eight were doctors, six were nurses, twelve were nursing technicians, three were nursing assistants, three were physiotherapist, making a total of 32 health professionals. Data were collected based on a key question: What is the meaning of humanization concerning medical assistance for you? The data collected were confirmed through systematic observation and the answers of the subjects, then constituting three theme categories: physical and emotional comfort and professional commitment.

\section{Results}

About the emotional comfort subject, speeches revealed that prescription and administration of medicines is indispensable to the treatment, but it is also necessary to take care of the patient with special attention, dedication, and respect him as a human being taking into account his beliefs, values, wishes, expectations concerning the treatment and evolution of his state of health. Those 


\section{* Nursing Abstracts from the 2nd Conference of the World Federation of Critical Care Nurses:}

who were interviewed considered that the health team should seek for alternatives to improve the assistance, taking into consideration not only technical matters but also personal values as well as learning about the understanding of the real meaning of human care. About the physical comfort subject, the necessary steps quoted were: never leave the patient alone; always check the installation of the ventilating prosthesis; assess and control pain in the patient; pay attention to the position and functioning of catheter; aspire secretions whenever necessary; care about the frequent mobilization of the patient; check and register the characteristics of secretions; perform body hygiene.

\section{Conclusions}

We noticed that the major focus of concern of health professionals who work at an ITU is caring with physical comfort. About the professional commitment subject it was outstanding the fact that professionals seek for the excellence of assistance and for this it is necessary to value people at work. Heath professionals who work at an ITU should consider in their everyday activities new perspectives about what to do for their profession implies a commitment to the patient to be present whenever possible assisting his questions and disturbances, which corresponds to continuous dedication to the never ending act of doing concerning the desire to overcome death by making use of solidarity and ethics.

\section{NEWBORN THERMAL REGULATION: A NURSERY PROTOCOL}

MF Diz1 ${ }^{1}$, CL Camargo ${ }^{2}$, MTA Calasans ${ }^{2}$, TF Batista ${ }^{1}$, NS Olivei$\mathrm{ra}^{1}$, TSM Barsosa ${ }^{2}, \mathrm{MS}$ Araujo ${ }^{1}$, T Oliveira ${ }^{1}$, A Ribeiro $^{1}, \mathrm{G} \mathrm{Rego}^{1}$, NCC Melo ${ }^{2}, C M L$ Valente ${ }^{2}$

1 Faculdade de Tecnologia e Ciências- Salvador-Bahia-Brasil;2 Escola de Enfermagem da Universidade Federal da Bahia-SalvadorBahia-Brasil

\section{Background}

Although newborns are homoeothermic, they have restricted thermal stabilization because of their large body surface area when compared to their mass, limited subcutaneous lipid deposition to provide isolation, motorvascular instability and decreased metabolic capacity. The heat loss, which begins at delivery through the mechanisms of evaporation, conduction, radiation and convection, may continue during the newborn internment if a neutral environment is not provided, contributing to hypothermia and consequently to a weight gain retard, metabolic alterations, and as severe cases as nervous system depression with damage, most of the time, irreversible.

\section{Objectives}

To elaborate a nursery care protocol to maintain a suitable thermal-regulation for the high risk of newborn babies.

\section{Methods \\ A bibliographic study has been initiated about the topic and lately neonatal care nurses with intensive care experience have discussed in order to obtain theoretic and practical subsidies to elaborate the proposed protocol.}

\section{Results}

A nursery care protocol to the newborn at risk to thermal instability has been elaborated considering gestational age, newborn weight, type of bed, seriousness, multiple procedure needs, clothing and temperature control frequency. The implementation process count- ed on workshops to guarantee the nursery staff to know the utilized thermal regulation methods.

\section{Conclusions}

After the nursery thermal regulation protocol activities were tested and observed in a nursery environment, the results indicated that newborns presented lower rate of hypothermia in comparison to care provided prior to the protocol implementation. Observed results confirm that thermal regulation management have contributed to treatment and development of the newborns.

\section{O617 A PROPOSAL FOR INSERTION AND MAINTENANCE OF CENTRAL VEIN CATHETER OF PERIPHERAL INSER- TION PROTOCOL}

MF Diz1, CL Camargo2, TF Batista1, NCC Melo2, MTA Calasans2, CML Valente2, MS Araujo1, NS Oliveira1, TSM Barbosa1, T Oliveira1, G Rego1, A Ribeiro1

1 Faculdade de Tecnologia e Ciências-Salvador-Bahia-Brasil;2 Escola de Enfermagem da Universidade Federal da Bahia-Salvadorbahia-Brasil

\section{Background}

The central vein catheter of peripheral insertion (CCPI) is a display inserted peripherally mainly in veins that are centrally located. This display is indicated when patient need intravenous solution for long periods of time and/or medication infusions. Its use has been increased in neonatal intensive care units because of its extended permanence period since it allows intravenous infusion of hyperosmolar and irritating solutions without compromising newborns cutaneous integrity, it decreases the risk of infection and mechanical complications and avoids surgical procedures for the placement of other catheters. This is an invasive procedure that puts the patient into risk, but it can be decreased by infection control using maximum barrier techniques, continue education programs, quality control achieved by two trained and qualified nurses and/ or doctors. The technical and legal responsibility of nurses insertion and manipulation in CCPI in Brazil is standardized by law 7498/86, decree 94406/87, under Nurse Federal Council number 240/2000 according to Health Ministry decree number 272 on April 8th 1998.

\section{Objectives}

The aim of this study was to standardize the nursery care at the CCPI insertion and maintenance in order to minimize complications, avoid excessive newborn manipulation, decrease the infection rate and guarantee safe venous fit. Methods

A bibliographic study has been initiated about the topic and lately neonatal care nurses with intensive care experience have discussed about it in order to elaborate the proposed protocol.

\section{Results}

The nursery protocol to CCPI insertion and maintenance was elaborated considering the following aspects: use indication, inclusion and exclusion criterions, peripheral fit options to insert the catheter, time of permanence, complications signs, dressing standardization and catheter handling.

\section{Conclusions}

With the implementation of the purposed protocol, the results indicated that complications rate related to the catheter utilization decreased and an increase of the catheter permanence period and reduction in venous dissection at the unit. 


\section{RELATIONSHIP BETWEEN CRITICAL PATIENT'S RELATIVES; RELATIONSHIP ICU NURSE - RELATIVES AND ITS INFLUENCE IN THE ABOVE MENTIONED RELA- TION \\ N Gómez, O Ortega \\ Intensive Care Unit of the University Insular Hospital of Gran Ca- naria (Canarias-España)}

\section{Background}

In spite of the development and the technological innovation that allows to offer a better welfare quality, the hospital admission of patients generate an uproot from their home and an emotional impact in their relatives, that begins just in the moment in which the patient is admitted in the hospital and is further accentuated as they enter into the Intensive Care Unit (ICU). Family members who suffer for a critically ill relative are usually immersed in atmosphere of insecurity, need and crisis and it is likely that they will need to be understood, before being helped. To become closer to the person in crisis, it is necessary to focus the attention in the art of communication, being able to speak and to be listened to achieve emotional discharge.

\section{Objectives}

1. To identify and to analyze the relationship among relatives before entering the ICU, 2. To define the influence that the nurse - relative relationship has in the above mentioned relation, 3 . To analyze the nurse - relative relationship.

\section{Methods}

Extracted from a study pilot made in the ICU of the University Insular Hospital of Gran Canaria (3rd Level), a transverse descriptive-observational study was designed. The extraction of information was made using two questionnaires of our own elaboration (one for nurses and another one for relatives), during three months. For statistical analysis, a Statistical Package for the Social Sciences (v12.0) was used. $\mathrm{P}<0.010$ was considered statistical significant

\section{Results}

The relatives who met each other before the patient entered the $\mathrm{ICU}$, felt helped, understood, and the above mentioned relationship helped them to understand the disease of the relative. Regarding the kind of help that the relatives offered to each other, "it helped them better to carry through the pathological process of the relative" and "created friendship" correspond to the major percentages of answer. The fact that nurses had a relationship with relatives of less than 10 minutes is associated to the relationship established between the relatives before entering in the ICU, they felt helped, understood, and the above mentioned relationship helped them to understand the disease of the relative, this was a fact that did not happen when the nurses spoke more than 10 minutes with relatives.

\section{Conclusions}

1. When the relatives had a relationship among them before entering in the ICU, they felt helped, understood, and the above mentioned relationship helped them to understand the disease of the relative. 2 . From the above mentioned interrelationship sprouted a support that help them to carry through the best possible way in order to face the pathological process of the patient and friendship to be born. 3. The interrelationship among critically ill patient's relatives was due to the fact that nurses were related to them for less than 10 minutes. 4 . Both the nurses and the relatives lacked of time for building a relationship.

\section{INCIDENCE OF PRESSURE ULCERS IN NEUROIN- TENSIVE CARE UNIT}

S Diccini, C Camaduro, ARC Bettencourt

Federal University of São Paulo

\section{Background}

Patients with spinal cord injuries, neurologic impairment, or advanced age are at high risk for pressure ulcers.

\section{Objectives}

The aim of the study was to determine the incidence of pressure ulcers in neurosurgical patients during the postoperative period in the neurointensive care unit (NICU).

\section{Methods}

A prospective follow-up study was conducted in a neurointensive care unit of a university hospital located in the city of São Paulo, Brazil, in the period of November, 2002 to May, 2003. Patients with preoperative pressure ulcers were excluded from the study. The skin of patients was observed daily during the postoperative period upon discharge. The Braden Scale was used to assess risk and the pressure ulcers were classified into four stages (Stage I, II, III and IV). Results

Thirty-eight patients were enrolled, 24 (63.2\%) females and 14 $(36.8 \%)$ males, and the mean age was 49 years (range 20 to 78 ). The reason for the operation was brain tumor (26 patients), cerebral aneurysms ( 6 patients), arterio-venous malformations (4 patients), spinal cord tumor ( 1 patient) and spinal cord injury ( 1 patient). On admission in the NICU, thirty-four (89.5\%) patients had Braden scores ranging from 8 to 16 . Six (15.8\%) patients of the 38 subjects developed pressure ulcers during the study, $4(66.7 \%)$ females and $2(33.3 \%)$ males. Of these, $3(50 \%)$ patients developed a Stage I ulcers, 2 (33.3\%) developed a Stage II ulcers, and 1 (16.7\%) developed a Stage IV ulcer. No one developed a Stage III ulcers. Pressure ulcers developed primarily on the malleolus $(33.3 \%)$, heels $(33.3 \%)$, ischium $(16.7 \%)$ and back of the head $(16.7 \%)$. The Braden Scale had scores ranging from 8 to 14, when the patients developed pressure ulcers. The pressure ulcers developed between 11 and 55 days of NICU admission.

\section{Conclusions}

The incidence of pressure ulcers in NICU was $15.8 \%$ and these results increase the challenge to nurse staff in the preventive care on the neurosurgical patients.

\section{O660 MEASURING NURSING WORKLOAD TO VERIFY NURSE: PATIENT RATIO IN A CARDIAC SURGERY INTEN- SIVE CARE UNIT}

AJ Ducci1, IY Whitaker2

1 Hospital São Paulo, São Paulo, Brazil;2 Universidade Federal De São Paulo - São Paulo- Brazil

\section{Background}

The instruments used for measuring nursing workload in the intensive care unit (ICU) has helped nurses to manage the nurse:patient ratio, in order to reach high quality of nursing care. 


\section{Objectives}

The aims of this study were to measure nursing workload in a cardiac surgery ICU using NAS (Nursing Activities Score), TISS-28 (Therapeutic Intervention Scoring System-28) and NEMS (Nine Equivalents and nursing Manpower and use Score) and, to verify nursing staff: patient ratio determined by nursing workload index and daily schedule of nursing staff in ICU.

\section{Methods}

This descriptive study was conducted in a cardiac surgery ICU of the University Hospital in São Paulo, Brazil. The data related to adult patients underwent cardiac surgery, who stayed in ICU at least 24 hours, from October to November 2004, were gathered prospectively. The scores TISS-28, NEMS and NAS were collect every day, from admission to discharge of the ICU. The number of nursing staff in each shift was obtained from the nursing schedule planning.

\section{Results}

Among the fifty five patients included in this study, $52 \%$ were male and the mean age was 62,7 years (19-85; SD 12,9). The length of stay average was four days (1-69; SD 9,6) and the mortality rate was $7,3 \%$. Admissions from operation room were predominant $(61,82 \%)$. Two hundred and eighty three measures of TISS-28, NEMS and NAS were collected and the average scores were, respectively, 25,4 points, 24,3 points and 66,4\%. Daily analysis of three instruments showed the NEMS average score was lower than TISS-28 on both months, but it was not statistically different. In a shift of six hours, the workload of staff nursing used almost four hours to take care of only one patient or $67 \%$ of total period of work. The average score of NAS was $66,4 \%$ and the need of take care of patient used from $53,7 \%$ to $72,8 \%$ of time in six hours shift. In the morning shift was observed higher staff nursing: patient ratio and in the afternoon shift the lowest ratio. The staff nursing/ patient ratio decreased on weekend and holiday. According to the index it was necessary to improve nursing staff of afternoon and night shift.

\section{Conclusions}

The instruments applied in this study showed that the average time to take care one patient indicates a nursing staff: patient ratio of $1: 1$. In addition, this study showed that the three instruments were useful to describe real nursing workload in cardiac surgery ICU. 